\title{
Geometry of quantum complexity
}

\author{
Roberto Auzzi $\odot,{ }^{1,2, *}$ Stefano Baiguera $\odot,{ }^{3, \dagger}$ G. Bruno De Luca $\odot,{ }^{4, \$}$ Andrea Legramandi $\odot,{ }^{5,}$ \\ Giuseppe Nardelli®, ${ }^{1,6, \|}$ and Nicolò Zenoni $\oplus^{1,2,7, \uparrow}$ \\ ${ }^{1}$ Dipartimento di Matematica e Fisica, Università Cattolica del Sacro Cuore, \\ Via Musei 41, 25121 Brescia, Italy \\ ${ }^{2}$ INFN Sezione di Perugia, Via A. Pascoli, 06123 Perugia, Italy \\ ${ }^{3}$ The Niels Bohr Institute, University of Copenhagen, Blegdamsvej 17, DK-2100 Copenhagen Ø, Denmark \\ ${ }^{4}$ Stanford Institute for Theoretical Physics, Stanford University, Stanford, California 94306, USA \\ ${ }^{5}$ Department of Physics, Swansea University, Swansea SA2 8PP, United Kingdom \\ ${ }^{6}$ TIFPA-INFN, clo Dipartimento di Fisica, Università di Trento, 38123 Povo (TN), Italy \\ ${ }^{7}$ Instituut voor Theoretische Fysica, KU Leuven, Celestijnenlaan 200D, B-3001 Leuven, Belgium
}

(Received 30 November 2020; accepted 23 April 2021; published 19 May 2021)

\begin{abstract}
Computational complexity is a quantum information concept that recently has found applications in the holographic understanding of the black hole interior. We consider quantum computational complexity for $n$ qubits using Nielsen's geometrical approach. In the definition of complexity there is a big amount of arbitrariness due to the choice of the penalty factors, which parametrizes the cost of the elementary computational gates. In order to reproduce desired features in holography, such as ergodicity and exponential maximal complexity for large number of qubits $n$, negative curvatures are required. With the simplest choice of penalties, this is achieved at the price of singular sectional curvatures in the large $n$ limit. We investigate a choice of penalties in which we can obtain negative curvatures in a smooth way. We also analyze the relation between operator and state complexities, framing the discussion with the language of Riemannian submersions. This provides a direct relation between geodesics and curvatures in the unitaries and the states spaces, which we also exploit to give a closed-form expression for the metric on the states in terms of the one for the operators. Finally, we study conjugate points for a large number of qubits in the unitary space and we provide a strong indication that maximal complexity scales exponentially with the number of qubits in a certain regime of the penalties space.
\end{abstract}

DOI: 10.1103/PhysRevD.103.106021

\section{INTRODUCTION}

An important problem in theoretical quantum computation is to determine the best quantum circuit to implement a desired unitary transformation. In general, this might be a challenging question. Moreover, it would be nice to have better theoretical tools to prove if a quantum computation problem has or not an efficient solution. The concept of quantum computational complexity has been introduced to answer these questions. Complexity itself is defined in a

\footnotetext{
roberto.auzzi@unicatt.it

†stefano.baiguera@nbi.ku.dk

†gdeluca@stanford.edu

\$andrea.legramandi@swansea.ac.uk

"giuseppe.nardelli@unicatt.it

nicolo.zenoni@unicatt.it
}

Published by the American Physical Society under the terms of the Creative Commons Attribution 4.0 International license. Further distribution of this work must maintain attribution to the author(s) and the published article's title, journal citation, and DOI. Funded by SCOAP ${ }^{3}$. rather heuristic way as the minimal number of computational gates required to build a given unitary operator with some tolerance. In order to improve the quantitative understanding, a geometrical approach to computational complexity in quantum mechanics was introduced in [1] and further studied in [2-5]. The basic idea is to introduce a Riemannian metric in the space of unitary operators acting on a given number of qubits, which quantifies how hard it is to implement a given quantum computational task. The distance induced by the metric in the space of unitary operators is used as a measure of the complexity of the quantum operation.

An additional motivation to study complexity arises from the desire of understanding the physics of the black hole interior [6-10]. Quantum information theory already provided us with many insights along the road to understand quantum aspects of gravity. This is especially powerful in the framework of AdS/CFT. The concept of entanglement entropy has a natural dual in terms of area of extremal surfaces [11]. Recently, such a geometric realization of entanglement led us to a better understanding of the Page curve [12] for an evaporating black hole, see, e.g., [13-15]. 
It is natural to conjecture that other features of holographic spacetime are encoded in other quantum information quantities, such as complexity. In the context of AdS/CFT correspondence, the growth of computational complexity was proposed as the boundary dual of the growth of the size of the Einstein-Rosen bridge connecting the left and the right sides of an eternal black hole in antide Sitter spacetime. Two main holographic duals for complexity were proposed:

(i) The complexity = volume conjecture relates complexity to the volume of an extremal slice anchored to the boundary [6-8];

(ii) The complexity = action conjecture relates complexity to the action computed in the WheelerDeWitt patch [16,17].

Holographic complexity was recently studied in a large variety of settings, see, e.g., [18-25]. Another promising generalization is provided by subregion complexity [26-39]. The appropriate notion of complexity in quantum field theory, dual to these holographic quantities, is still an open problem. One of the most promising and challenging approaches is to generalize Nielsen's geometric method to quantum field theory, see, e.g., [40-53].

A conjecture about the generic time evolution of complexity has been proposed in [9]. In this picture, at early times complexity grows linearly for a period that is exponential in the number of qubits $n$. This initial phase is called the complexity ramp. At time $t \propto e^{n}$ it reaches its maximum value and then it flattens for a very long time $t \propto e^{e^{n}}$, doubly exponential in $n$ (this is called the complexity plateau). After this very long time, quantum recurrence can bring back the system to subexponential values with non-negligible probability. This picture, if confirmed, would give us interesting insights on the quantum history of black holes. For instance, white holes could be thought of as the gravity duals of a phase of decreasing complexity due to quantum recurrence.

The geometrical approach by Nielsen is an interesting direction to put the definition of complexity on firmer grounds. There is an important order zero property that complexity must satisfy in order to fit the expectations in [9]: in the limit of large number of qubits $n$, the maximal complexity should scale exponentially with $n$.

A full understanding of complexity is still an open problem already in quantum mechanics. In particular, there are many ways to define geometric computational complexity. Riemannian geometry is just a possibility. It could be that Finsler geometry is more appropriate to investigate complexity, both for quantum computer science [1] and in the holographic case [47]. Even in the more traditional paradigm of Riemannian geometry, there is a lot of ambiguity in defining complexity. Part of it comes from the choice of the penalty factors for the Hermitian generators of the unitary transformations, which implement the physical concept that some operations can be harder than others to perform in a quantum circuit. The simplest possibility would be to choose a uniform penalty factor, independent of the number of qubits entangled by the given quantum operation. However this brings to a maximal allowed complexity which does not scale exponentially with the number of qubits [1] and so it does not match our expectations. It was suggested in [1] that Finsler metrics with uniform penalty factors or Riemannian metrics with nonuniform penalties may instead give an exponential complexity in some regions of the parameter space.

An interesting toy model for many desired features of complexity geometry was proposed in [54], considering geodesics in a compact two-dimensional space with negative curvature. In particular, it was argued that negative curvature gives an interesting crossover between $L^{2}$ norm at small distances and an effective $L^{1}$ norm at large distances. This allows us to remain in the framework of Riemannian geometry, which is easier to deal with compared to Finsler geometry.

Another desirable property of complexity metric is the ergodicity of geodesics, which is important to apply thermodynamical arguments to complexity evolution [55-57]. Ergodicity in this context refers to the general idea that the trajectory of a generic state along a geodesic will eventually visit all the allowed portions of the unitary space. There are classical mathematical results (see, e.g., [58]) showing that the geodesic flow on a manifold with all negative sectional curvatures is ergodic. The complexity metric with uniform penalty factors is positively curved in all the directions and does not have an ergodic geodesic flow. The introduction of nonuniform penalty factors can make some of the sectional curvatures negative [5], but not all of them. If the negative contribution dominates, we expect that the geodesic motion is still ergodic.

Let us denote with $w$, which we will refer to as the weight, the number of qubits that are simultaneously entangled by a given generator. In [5], the following choice of penalty factors was studied in detail for systems of $n$ qubits:

$$
\begin{array}{ll}
q(w)=1, & w \leq 2, \\
q(w)=q, & w>2 .
\end{array}
$$

In order to get negative scalar curvature, a penalty factor $q$ of order $4^{n}$ is needed. This brings to a singular limit where the negative scalar curvature is dominated by a few negative sectional curvatures that diverge in the large $n$ limit. The penalty choice in (1.1) was called draconian in [55]. It was argued that this choice is not appropriate to reproduce black hole properties such as scrambling time and switchback effect [59].

For this reason, in [55], a less drastic choice of penalty factors was advocated. In this paper we will study a variant of this choice: 


$$
q(w)=\alpha^{w-1},
$$

where $\alpha>1$ is a constant. We will call the choice (1.2) progressive penalties. In order to understand complexity geometry in an analytic way, we will propose a large $\alpha$ limit in which complexity geometry might be studied order by order in the expansion parameter $\alpha^{-1}$. The leading order sectional curvatures scale as $\alpha^{0}$. We find closed form for all the curvatures up to the next-to-leading order $\alpha^{-1}$.

As recently emphasized in [60], two different but strongly related definitions of complexity can be considered for quantum systems:

(i) Unitary complexity quantifies how hard it is to build some unitary operators. It was physically motivated by the problem of quantum circuit computational complexity [1-5].

(ii) State complexity quantifies how hard it is to build a unitary transformation that transforms the reference state to the target state $[6-8,10]$. This is the most natural way to apply the notion of complexity to holography.

For $n$ qubits, the unitary complexity metric is defined on the group manifold $S U\left(2^{n}\right)$ and it is a homogeneous but not isotropic metric. In particular, homogeneity tells us that scalar quantities (such as curvature) are constant. The state complexity metric instead is defined on $\mathbb{C P}^{2 n-1}$ and it is neither isotropic nor homogeneus. The number of dimensions is smaller than in the unitary metric, but the geometrical structure is more complicated, because this space is not homogeneous and the scalar curvature is not constant. In this paper we point out that the relation between unitary and states complexity is a particular case of Riemannian submersion [61]. For this reason, geodesics on the state space are determined by just projecting a class of geodesics on the unitary space, the horizontal ones [62]. Moreover, the curvatures in the state space can be obtained from the curvatures in the unitary space by O'Neill's formula [61].

Complexity is determined (both in unitary and state spaces) as the length of the shortest geodesic which connects two given points. Given a geodesic starting from an initial point $P$, there exists another point along the geodesic where it begins to fail to be the minimal one. This is called the cut point of the geodesic. The cut locus of a given point $P$ is defined as the set of all the cut points of the geodesics starting from $P$. For unitaries complexity, the metric is homogeneus and then it is enough to study the cut locus at the identity. In general, finding the cut locus is a complicated problem. A useful approach is to consider conjugate points which, roughly speaking, are the points of the manifold that can be joined by a continuous oneparameter family of geodesics. From a general result in geometry, we know that a given geodesic fails to be the minimizing one after its first conjugate point. The converse is not true: a geodesic may stop to be minimizing well before a conjugate point is reached. In this paper we study conjugate points of complexity metric both for one and for a large number of qubits. From this analysis, we find an evidence that maximal complexity scales exponentially with $n$ in the progressive model for large $\alpha$.

The paper is organized as follows. In Sec. II we review some results of [5] for the complexity geometry in the unitary space for an arbitrary number of qubits and we derive a useful explicit formula for sectional curvatures. In Sec. III we briefly discuss some few qubits examples. In Sec. IV we consider the situation of a large number of qubits $n$ : after a brief review of the draconian case, we study the progressive choice of penalties (1.2). In Sec. V we discuss state complexity and we point out the relevance of the Riemannian submersion, which relates the geometry of the states to the one of the unitaries. We also derive a closed-form expression for the state metric. In Sec. VI we study the conjugate points in the unitary space of a simple class of geodesics, given by the exponential of the generators which are eigenvalues of the penalty matrix. We conclude in Sec. VII. Technical details and examples are deferred to appendices.

\section{UNITARY COMPLEXITY}

We will first review several useful results about the geometry of unitary complexity, following [5]. We will consider the space of unitary operators acting on a $n$ qubits system, which is $S U\left(2^{n}\right)$. The tangent vector at a generic point $U_{0}$ can be specified in terms of a traceless Hermitian generator $H$, which is the tangent to the curve

$$
U(t)=e^{-i H t} U_{0},
$$

evaluated at $t=0$.

For a generic curve $U(t)$ in the space of unitaries determined by the Schrödinger equation $\dot{U}(t)=-i H(t) U(t)$, we can define in general a complexity norm using a suitable Riemannian metric:

$$
l=\int d t\langle H(t), H(t)\rangle^{1 / 2}
$$

In our application, we will consider $\langle\ldots\rangle$ to be a positivedefinite inner product independent of the group point $U$. Such a metric can be therefore defined at the origin of the group manifold and it can be mapped to every point of the manifold using right translations. This metric is usually called a right-invariant metric $[63,64]$ and can be defined starting from a given scalar product at the origin:

$$
\langle H, K\rangle=\frac{\operatorname{Tr}[H \mathcal{G}(K)]}{2^{n}} .
$$

Here $\mathcal{G}$ is a positive-definite operator on the space of unitaries, i.e., a superoperator. This terminology is common in the quantum information literature. 


\section{A. Comments on the choice of basis}

We work with the basis defined by generalized Pauli matrices $\sigma$, which are nothing but the tensor products of $n$ matrices, each of which can be either a $S U(2)$ Pauli matrix $\sigma_{i}(i=1,2,3)$ or the identity $\mathbb{1}_{2}$. We define the weight $w(\sigma)$ as the number of $S U(2)$ Pauli matrices involved in the tensor product $\sigma$. We will consider only diagonal metrics in our basis, i.e., $\mathcal{G}(\sigma)=q_{\sigma} \sigma$, so that the inner product (2.3) reads

$$
\langle\sigma, \tau\rangle=q_{\sigma} \delta_{\sigma \tau},
$$

and we denote by $q_{\sigma}$ the penalty factor for the generator $\sigma$ normalized as $\operatorname{Tr}\left(\sigma^{2}\right)=2^{n}$. We call the choice $q_{\sigma}=1$ the unpenalized choice.

The generalized Pauli matrices have a useful property: if we choose two elements of the basis, they either commute or anticommute. In the one qubit case this follows directly from the Pauli matrices algebra and it can be easily generalized to the $n$ qubits case. In particular, let us consider the product $\tau \sigma$ of two generalized Pauli matrices. Then we have

$$
\sigma \tau=(-1)^{l} \tau \sigma,
$$

where $l$ is the number of the corresponding entries in the tensor products in $\tau$ and in $\sigma$ involving different Pauli matrices.

It is useful to count the number of generalized Pauli matrices anticommuting with a given $\sigma$. If $\sigma=\mathbb{1}$, trivially there are no operators anticommuting with it. If $\sigma \neq \mathbb{1}$, a generalized Pauli matrix $\rho$ anticommutes with it under the condition that there is an odd number $l$ of corresponding entries in the tensor products in $\sigma$ and $\rho$ involving different Pauli matrices. Let us suppose that $\sigma$ has weight $w$ (its tensor product contains $w$ Pauli matrices). Then, we necessarily have $0 \leq l \leq w$. Among the $n$ entries of the tensor product in $\rho$, the $n-w$ entries in correspondence with the identity $\mathbb{1}_{2}$ in $\sigma$ can arbitrarily be any matrix in the basis $\left(\mathbb{1}_{2}, \sigma_{i}\right)$ indifferently. Thus we have $4^{n-w}$ choices for such entries. For the remaining $w$ entries of $\rho$, we have $\left(\begin{array}{c}w \\ l\end{array}\right)$ choices for the $l$ positions of the unequal Pauli matrices. Once this is fixed, there is a further $2^{w}$ degeneracy of choices. Summarizing, the number of generalized Pauli matrices $\rho$ anticommuting with $\sigma$ is

$$
4^{n-w} \sum_{l \text { odd }=1}^{w}\left(\begin{array}{c}
w \\
l
\end{array}\right) 2^{w}=\frac{4^{n}}{2}
$$

It is remarkable that the number of $\rho$ anticommuting with a given $\sigma \neq \mathbb{1}$ does not depend on the weight of $\sigma$.

The commutator of two elements of the basis (if not vanishing) is proportional to another element of the basis, because the two products in the commutator give the opposite matrix ( $l$ is odd). Given two noncommuting elements of the basis $\sigma$ and $\tau$, we define $q_{[\sigma, \tau]}$ as the penalty of their commutator; if $[\sigma, \tau]=0$ we set by definition $q_{[\sigma, \tau]}=1$.

\section{B. Connection and geodesic equation}

Let us now derive an expression for the Levi-Civita connection $\nabla$ compatible with the metric (2.3). This is given by the Koszul formula [65], which, thanks to the fact that the inner product can be computed at the identity (and therefore is constant in a suitable basis), simplifies to

$-2 i\left\langle\nabla_{X} Y, Z\right\rangle=\langle[X, Y], Z\rangle+\langle[Z, X], Y\rangle-\langle[Y, Z], X\rangle$,

where $X, Y, Z$ are right-invariant fields interpreted as Hermitian matrices at the origin. Equation (2.7) allows us to define

$$
\nabla_{X} Y=\frac{i}{2}\left([X, Y]+\mathcal{G}^{-1}([X, \mathcal{G}(Y)]+[Y, \mathcal{G}(X)])\right) .
$$

Setting $Y=X$ in Eq. (2.8), we obtain the geodesic equation, which is nothing but the Euler-Arnold ${ }^{1}$ equation [64]:

$$
\dot{X}+i \mathcal{G}^{-1}([X, \mathcal{G}(X)])=0 .
$$

In general we expect that geodesics have an intricate behavior. Eq. (2.9) shows that there exists a simple class of geodesics, given by the exponential of an eigenvector of the penalty operator $\mathcal{G}$. We will call the geodesics which are exponential of such eigenvectors "exponential geodesics." We study the behavior of their conjugate points in Sec. VI.

\section{Riemann tensor}

Let us now specialize the discussion to $S U\left(2^{n}\right)$ using Pauli matrices $\rho, \sigma, \tau, \mu$, which can be viewed as rightinvariant frame fields. The curvature tensor is [5]

$R_{\rho \sigma \tau \mu}=\left\langle\nabla_{\rho} \tau, \nabla_{\sigma} \mu\right\rangle-\left\langle\nabla_{\sigma} \tau, \nabla_{\rho} \mu\right\rangle-\left\langle\nabla_{i[\rho, \sigma]} \tau, \mu\right\rangle$.

Using Eq. (2.8), we find

$$
\nabla_{\sigma} \tau=i c_{\sigma, \tau}[\sigma, \tau], \quad c_{\sigma, \tau} \equiv \frac{1}{2}\left(1+\frac{q_{\tau}-q_{\sigma}}{q_{[\sigma, \tau]}}\right) .
$$

The Riemann tensor is given by the expression:

$$
\begin{aligned}
R_{\rho \sigma \tau \mu}= & c_{\rho, \tau} c_{\sigma, \mu}\langle i[\rho, \tau], i[\sigma, \mu]\rangle-c_{\sigma, \tau} c_{\rho, \mu}\langle i[\sigma, \tau], i[\rho, \mu]\rangle \\
& -c_{[\rho, \sigma], \tau}\langle i[i[\rho, \sigma], \tau], \mu\rangle .
\end{aligned}
$$

\footnotetext{
${ }^{1}$ Recent applications of the Euler-Arnold equations in relation to complexity were found in $[52,53]$.
} 
Since Eq. (2.12) depends just on commutators, the Riemann curvature of a subgroup of unitaries does not depend on the metric data outside this subgroup. For example, complexity on a one qubit subgroup depends just on penalties of generators acting on that particular subgroup. An important result [5] is that the component $R_{\rho \sigma \tau \mu}$ vanishes unless the product of the corresponding generalized Pauli matrices $\rho \sigma \tau \mu$ is proportional to the identity.

\section{Sectional curvatures}

The sectional curvature is defined as half of the scalar curvature of a two-dimensional submanifold with tangent space specified by the directions $(\rho, \sigma)$. The general expression for the sectional curvature of the plane determined by the vectors $(v, w)$ is [66]

$$
K(v, w)=\frac{R_{\alpha \beta \gamma \delta} v^{\alpha} w^{\beta} w^{\gamma} v^{\delta}}{\left(v_{\alpha} v^{\alpha}\right)\left(w_{\beta} w^{\beta}\right)-\left(v_{\alpha} w^{\alpha}\right)^{2}} .
$$

The quantity $K(v, w)$ depends just on the plane which is defined by $(v, w)$ and does not depend on their normalization. The sectional curvature is a nonlinear object and it is a nontrivial function of the orientation of the plane; in general, in order to determine $K$ on an arbitrary plane it is not enough to determine it on the planes defined by couples of vectors on an orthogonal basis.

The generalized Pauli matrices are orthogonal but not normalized, see Eq. (2.4). The sectional curvature in the plane spanned by two generalized Pauli matrices is

$$
K(\rho, \sigma)=\frac{R_{\rho \sigma \sigma \rho}}{q_{\rho} q_{\sigma}} .
$$

From Eq. (2.12) we find

$$
\begin{aligned}
R_{\rho \sigma \sigma \rho}= & c_{\rho, \sigma} c_{\sigma, \rho}\langle i[\rho, \sigma], i[\sigma, \rho]\rangle \\
& -c_{[\rho, \sigma], \sigma}\langle i[i[\rho, \sigma], \sigma], \rho\rangle .
\end{aligned}
$$

This vanishes if $\rho$ and $\sigma$ commute. Instead, in the case of anticommuting $\rho$ and $\sigma$, a direct calculation gives

$$
\begin{aligned}
& \langle i[\rho, \sigma], i[\rho, \sigma]\rangle=4 q_{[\rho, \sigma]}, \\
& \langle i[i[\rho, \sigma], \sigma], \rho\rangle=-4 q_{\rho},
\end{aligned}
$$

where in both the relations we repeatedly used the fact that $\rho$ and $\sigma$ anticommute. We can also use the property $q_{[\rho, \sigma], \sigma]}=q_{\rho}$ to get the sectional curvature

$$
K(\rho, \sigma)=\frac{1}{q_{\rho} q_{\sigma}}\left[-3 q_{[\rho, \sigma]}+2\left(q_{\rho}+q_{\sigma}\right)+\frac{\left(q_{\rho}-q_{\sigma}\right)^{2}}{q_{[\rho, \sigma]}}\right],
$$

which is valid if $[\rho, \sigma] \neq 0$ (otherwise $K(\rho, \sigma)=0$ ).
This formula, which as far as we know is new and not contained in [5], has interesting consequences. We see that the only negative contribution to $K(\rho, \sigma)$ comes from $q_{[\rho, \sigma]}$ : $K$ can become negative only if the commutator $[\rho, \sigma]$ has a large enough penalty factor. In general, we expect that $K$ is positive, unless $q_{[\rho, \sigma]}$ is big enough compared to $q_{\rho}$ and $q_{\sigma}$.

One may wonder if it is possible to get negative all the sectional curvatures of the orthogonal basis. This is not possible, because the sectional curvatures of the one qubit subspace depend just on the one qubit penalty factors. In Sec. III A we will show that at least two out of three independent sectional curvatures are always positive for one qubit.

\section{E. Ricci tensor and curvature}

Sectional curvatures are related to Ricci tensor and Ricci curvature. As shown in [5], in our basis the only nonvanishing component of the Ricci tensor $R_{\sigma \tau}$ are the diagonal ones, with $\sigma=\tau$. Given an orthonormal basis $\left\{e_{k}\right\}$ with $k=1, \ldots, N$ and such that $e_{1}=v$, we have the following result [66] valid for all Riemannian manifolds:

$$
R_{\alpha \beta} v^{\alpha} v^{\beta}=\sum_{k=2}^{N} K\left(v, e_{k}\right) .
$$

In this way the scalar curvature can be expressed in terms of the sectional curvatures as

$$
R=\sum_{k=1}^{N} R_{\alpha \beta} e_{k}^{\alpha} e_{k}^{\beta}=\sum_{\sigma, \rho} K(\rho, \sigma) .
$$

It should be emphasized that the sectional curvatures do not transform linearly as tensors, still their sum reproduces the Ricci scalar.

The sign of sectional curvatures plays a key role in relation to ergodicity [55]. Roughly speaking, the geodesic flow is called ergodic if its typical geodesic will eventually pass nearby to all the allowed portions of the operator space. The average of observables along the geodesic trajectory will then coincide with the average over the manifold of unitaries. In the context of the motion in the group manifold of unitaries, one can consider the time evolution of two neighboring geodesics intersecting at $t=0$ under infinitesimally close local Hamiltonians. In such a case, the deviation between the geodesics is governed by the sectional curvature corresponding to the section containing the two geodesics: if the sign is positive as in the standard inner product metric, then the geodesics converge. On the other hand, an appropriate choice of penalty factors allows to obtain negative sectional curvatures, implying that the geodesics diverge. The divergence of geodesics is an important requirement for quantum chaos, which in turn requires an ergodic behavior.

From a general theorem [58], we know that geodesic flow is ergodic in manifolds whose all sectional curvatures 
are negative. This result is not directly applicable to unitary complexity, because at least some of the sectional curvatures in the one qubit directions are always positive. Indeed, ergodicity of geodesic is still preserved in some examples where the curvature is partly negative and partly positive (see, e.g., [67]). In general, we expect that the presence of directions with mostly negative sectional curvatures is a strong indication of ergodic behavior of geodesics. From Eq. (2.19) we know that the scalar curvature is the sum of all the sectional curvatures of an orthogonal basis, and so we expect that negative scalar curvature $R$ is a detector of ergodicity. Unfortunately, we do not know about any rigorous mathematical theorem that relates the sign of $R$ to the ergodicity of geodesics.

In view of the investigation of conjugate points of the geodesics in Sec. VI, it is convenient to introduce a specific notation for the diagonal components of the Ricci tensor. Using an orthonormal basis $\{u(\sigma)\}$ in the algebra, we define

$$
\begin{aligned}
R_{\sigma}=R_{\alpha \beta} u^{\alpha}(\sigma) u^{\beta}(\sigma) & =\frac{R_{\sigma \sigma}}{q_{\sigma}}, \\
u^{\alpha}(\sigma) u^{\beta}(\sigma) g_{\alpha \beta} & =1 .
\end{aligned}
$$

Using the definitions for the curvature quantities given above, we start by considering in Sec. III the simple cases where the quantum-mechanical system is composed by one or two qubits. We will extract the sectional curvatures and the Ricci scalar and study their behavior in relation to various choices of the penalty factors on the generators. Then we will generalize in Sec. IV to the case with many qubits, where we will propose some choices of penalty factors to reproduce expected properties of complexity.

\section{FEW QUBITS EXAMPLES}

\section{A. One qubit}

Let us fix the penalty factor for $\sigma_{x}$ to 1 and denote the penalty factors for $\sigma_{y}$ and $\sigma_{z}$ by $Q$ and $P$. For $Q=1$, the metric has a $U(1)$ isotropic symmetry which rotates $\left(\sigma_{x}, \sigma_{y}\right)$. Applying the results of the previous section, the sectional curvatures of the planes selected by our orthonormal basis are

$$
\begin{aligned}
& K_{x y}=\frac{-3 P^{2}+2 P+2 P Q+Q^{2}+1-2 Q}{P Q}, \\
& K_{x z}=\frac{-3 Q^{2}+2 Q+2 P Q+P^{2}+1-2 P}{P Q}, \\
& K_{y z}=\frac{-3+2 P+2 Q+P^{2}+Q^{2}-2 P Q}{P Q},
\end{aligned}
$$

and the scalar curvature is

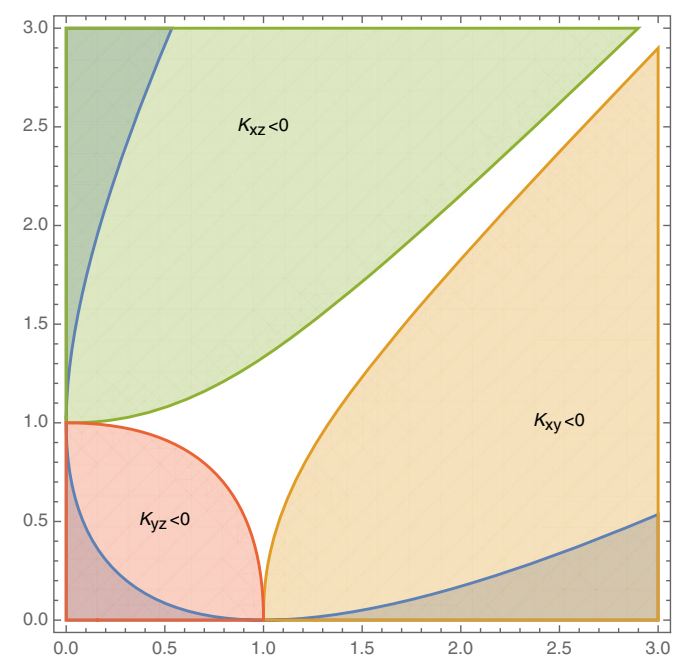

FIG. 1. Regions of negativity of sectional curvatures in the $(P, Q)$ plane. In the white region all the sectional curvatures are positive. The blue shaded regions correspond to a negative scalar curvature.

$$
R=-2 \frac{(Q-P)^{2}-2(P+Q)+1}{P Q} .
$$

The signs of sectional and scalar curvatures are shown in Fig. 1. Note that two out of the three sectional curvatures in Eq. (3.1) are positive in all the parameter space.

The sectional curvatures form a nonlinear object; these quantities are not enough to compute the sectional curvature in an arbitrary plane, which can be found using expressions from the Riemann tensor. In the one qubit case, we checked that the values in Eq. (3.1) correspond for all $P, Q$ to the maxima and minima of the sectional curvature.

Conventionally, we will call the generators with lowest penalty "easy" generators, and those with highest penalty "hard" generators. We are interested in limits where the maximal complexity becomes large, in general exponential in the number of states. So it might seem a contradiction to search for limits of large complexity in the one qubit Hilbert space. This is not necessarily the case: in order to explore a toy model with large maximal complexity, one may consider the limit where the weight factors $P, Q$ go to infinity.

One of these limits may be obtained by setting

$$
P=1, \quad Q \rightarrow \infty \text {. }
$$

In this case the scalar and the sectional curvatures diverge:

$$
\begin{aligned}
R & =8-2 Q, \quad K_{x y}=4-3 Q, \\
K_{x z} & =K_{y z}=Q .
\end{aligned}
$$

In general, if we set $P$ constant and we send $Q \rightarrow \infty$, we do not obtain a smooth limit. 
It is also interesting to consider the limit

$$
P=Q \rightarrow \infty \text {. }
$$

The scalar curvature remains small:

$$
\begin{aligned}
R & =\frac{8}{P}-\frac{2}{P^{2}}, \quad K_{x y}=K_{x z}=\frac{1}{P^{2}}, \\
K_{y z} & =\frac{4}{P}-\frac{3}{P^{2}} .
\end{aligned}
$$

In this case all the sectional curvatures are positive and become small.

Another possibility is to consider

$$
P=\beta Q \rightarrow \infty,
$$

with $\beta$ constant. At large $P$ we find that the sectional curvatures approach to constants. For $\beta \neq 1$, at large $P$ the scalar curvature is negative, $R=-2(\beta-1)^{2}$.

In all these limits the volume of the space (measured using the complexity metric) goes to infinity. From the point of view of complexity, instead, these limits are very different. In the case (3.3) the maximal complexity does not approach infinity, because the remaining easy generators are enough to build whatever unitary we want. Instead, in the cases in Eqs. (3.5) and (3.7) the maximal complexity goes to infinity, because the only easy generator at our disposal allows to produce just a very special class of unitary, i.e., the rotations along the $x$ axis.

\section{B. Two qubits}

The two qubits case is the simplest environment where we can address the question of what happens if one penalizes operators according to the number of qubits that are entangled at the same time.

We choose $A$ as penalty factor for the weight 1 matrices and $B$ as penalty factor for the weight 2 ones. The nonvanishing sectional curvatures $K(\rho, \sigma)$ in the orthonormal basis can take three values:

$$
a=\frac{1}{A}, \quad b=\frac{A}{B^{2}}, \quad c=\frac{4 B-3 A}{B^{2}} .
$$

The value $a$ arises when $(\rho, \sigma)$ have both weight $w=1$, the value $c$ when they have both $w=2$ and the value $b$ if they are generators with different weights. The multiplicity of each value of the sectional curvatures is

$$
N_{a}=12, \quad N_{b}=72, \quad N_{c}=36 .
$$

The scalar curvature is

$$
R=-12 \frac{3 A^{2}-12 A B-B^{2}}{A B^{2}}
$$

Let us specialize $A=1$ and $B=q$ with $q>1$. We are penalizing the weight 2 matrices (denoted as "hard") compared to the weight 1 matrices (denoted as "easy"). The scalar curvature is

$$
R=12 \frac{-3+12 q+q^{2}}{q^{2}},
$$

which is always positive. Note that in this case the structure of the algebra generators is as follows

[easy, easy $]=$ easy,$\quad[$ easy, hard $]=$ hard,

$[$ hard, hard $]=$ easy,

and so it gives rise to positive sectional curvatures, from Eq. (2.17). Although such a choice is the most intuitive, it necessarily provides positive curvatures, see also [60] for the same conclusion. Note that no singularity appears in the curvature if we send $q \rightarrow \infty$.

If we instead set $A=p$ and $B=1$, we are penalizing the weight 1 matrices and the scalar curvature is

$$
R=-12 \frac{3 p^{2}-12 p-1}{p} .
$$

Note that in this case the structure of the algebra generators is reversed

$[$ hard, hard $]=$ hard,$\quad[$ easy, hard $]=$ easy,

[easy, easy] $=$ hard,

and indeed gives negative curvature at large enough $p$, according to Eq. (2.17). This result gives a quantitative explanation of some intuitions discussed in [60].

We point out that the aforementioned case is not the only one where such a behavior occurs. In general, when we split the set of generators in two classes, one of which is a maximal subalgebra, the structure of commutators (3.12) and (3.14) always arises.

\section{MANY QUBITS}

We consider quantum systems composed by many qubits, which is the first step in the direction of a system with infinite degrees of freedom as it happens in field theory. In this case it is possible to study the dependence of the curvatures on the number of qubits, in order to understand the assignment of penalty factors that can reproduce physical phenomena like the switchback effect and scrambling.

The idea is to study the time evolution of complexity when the system of interest is subject to a perturbation. From the holographic point of view, this is usually performed with the introduction of a shock wave very far in the past, in such a way that the scrambling time 
corresponds to the delay after which the black hole reaches again the equilibrium [7]. From the perspective of quantum circuits, a useful model consists in the evolution of an epidemic [10]. If there is a single infected qubit that can interact with all the other ones via a local Hamiltonian, the scrambling time measures the scale after which the infection has involved a large enough number of qubits in order for complexity to reach the value $n$, corresponding to the number of qubits.

In this context, a related effect is the switchback one, which is a delay in the growth of complexity arising from cancellations between multiple shock waves or perturbations. Using the toy model introduced in [54], it was suggested that, in order to get a satisfying description of switchback effect and scrambling, the typical sectional curvatures should scale as $1 / n$ or $1 / n^{2}$ in the large number of qubits limit (depending on the variant of the model). For a recent discussion of the switchback effect for low number of qubits, see [68]. Even without restricting to a particular toy model, the divergence of sectional curvatures in the large number of qubits limit gives rise to a singular behavior that should be avoided. In this section we will study the consequences of various assignments of penalties on the behavior of curvatures.

Let us consider the case with $n$ qubits, equipped with a class of penalty factors that are functions just of the weight of the generators. Let us denote the penalty associated to the weight $k$ by $q_{k}$. The number of generalized Pauli matrices with weight $k$ in our basis is given by

$$
\mathcal{N}_{k}=3^{k}\left(\begin{array}{l}
n \\
k
\end{array}\right) \text {. }
$$

Given two generators $(\rho, \sigma)$, let us denote, respectively, by $M$ and $N$ the weights of $\rho$ and $\sigma$, and by $w$ the weight of the commutator $[\rho, \sigma]$. From the analysis given in Appendix A, we can show that $w$ can take the following values

$$
w_{r}=|M-N|+1+2 r,
$$

where the integer $r$ has the following range

$$
0 \leq r \leq \min (M, N)-1 .
$$

If two directions in the unitary space do not commute, the sectional curvatures can be obtained from Eq. (2.17), i.e.,

$$
K(M, N, r)=\frac{1}{q_{M} q_{N}}\left(-3 q_{w_{r}}+2\left(q_{M}+q_{N}\right)+\frac{\left(q_{M}-q_{N}\right)^{2}}{q_{w_{r}}}\right),
$$

where $K(M, N, r)$ denotes the sectional curvature of the plane spanned by generalized Pauli matrices of weights $M$ and $N$, whose commutator has weight $w_{r}$, given by
Eq. (4.2). We denote by $\mathcal{N}(M, N, r)$ the degeneracy of such sectional curvatures. We derive an explicit expression for $\mathcal{N}(M, N, r)$ in Appendix A.

If two directions commute $K(M, N, r)=0$; given a generalized Pauli matrix, about one half of the other Pauli matrices in the basis commute with it, see Eq. (2.6). So about one half of the total sectional curvatures vanish by construction, independently of the penalty factors.

\section{A. Draconian penalties}

The combination of 1 and 2 qubits operators is universal and can be used to build an arbitrary operator in $S U\left(2^{n}\right)$ [69]. This result suggests a somewhat minimal choice of penalty factors, studied in detail in [5]

$$
\begin{array}{ll}
q_{\sigma}=1, & w \leq 2, \\
q_{\sigma}=q, & w>2 .
\end{array}
$$

This choice does not distinguish different values of the weight $w>2$ and was called "draconian" in [60].

The sectional curvatures can be found using the general expression in Eq. (2.17), giving the values in Table I.

For $q=1$ we recover the case where all the penalty factors are equal, which corresponds to a bi-invariant metric on $S U\left(2^{n}\right)$. In this case all the nonvanishing sectional curvatures are equal and positive. The interesting region with negative curvature is at large $q$. So in this limit it makes sense to use the approximation where only the sectional curvatures at leading order in $q$ are considered.

Let us consider the approximation in which we keep just the $\mathcal{O}(q)$ and the $\mathcal{O}(1)$ terms. In this limit the only nonvanishing sectional curvatures are

$$
\begin{aligned}
& K(1,1,0)=K(2,1,0)=K(2,2,0)=1, \\
& K(3,2,0)=q, \\
& K(2,2,1)=4-3 q,
\end{aligned}
$$

with multiplicities

$$
\begin{aligned}
& \mathcal{N}(1,1,0)=6 n \\
& \mathcal{N}(2,1,0)=\mathcal{N}(2,2,0)=18 n(n-1), \\
& \mathcal{N}(3,2,0)=\mathcal{N}(2,2,1)=54 n(n-1)(n-2) .
\end{aligned}
$$

TABLE I. Values of the nonvanishing sectional curvature $K$ for various choices of $\rho, \sigma$ in the model with draconian penalty factors. We denote by $\mathcal{P}$ and $\mathcal{Q}$ the set of generators with $w \leq 2$ and $w>2$, respectively.

\begin{tabular}{lcc}
\hline \hline & {$[\rho, \sigma] \in \mathcal{P}$} & {$[\rho, \sigma] \in \mathcal{Q}$} \\
\hline$\rho, \sigma \in \mathcal{P}$ & $K=1$ & $K=4-3 q$ \\
$\rho, \sigma \in \mathcal{Q}$ & $K=\frac{4 q-3}{q^{2}}$ & $K=\frac{1}{q}$ \\
$\rho \in \mathcal{P}, \sigma \in \mathcal{Q}$ & $K=q$ & $K=\frac{1}{q^{2}}$ \\
\hline \hline
\end{tabular}


The scalar curvature then is

$$
R=-54 n(n-1)(n-2) q+6 n\left(36 n^{2}-99 n+64\right)
$$

This calculation is in agreement with the exact result computed in [5] in a different way:

$$
\begin{aligned}
R= & -54 q n(n-1)(n-2)+6 n\left[36 n^{2}-99 n+64\right] \\
& +\frac{1}{q}\left[\frac{4^{n}}{2}\left(4^{n}-1+\frac{3 n(3 n-1)}{2}\right)-6 n\left(45 n^{2}-117 n+74\right)\right]+ \\
& -\frac{1}{q^{2}}\left[3 n(3 n-1) 4^{n-1}-6 n(3 n-4)(6 n-7)\right] .
\end{aligned}
$$

In order to get negative curvature, we need $q \propto 4^{n}$ or larger. This means that $q$ has to grow exponentially with $n$. In particular, in this regime the scalar curvature is dominated by a small number (polynomial in $n$ ) of sectional curvatures whose magnitude grows like $|K| \approx q \approx 4^{n}$. This is a singular limit, and, as discussed in [55], this brings us to some unwanted properties in the scrambling and switchback effect of black holes complexity.

\section{B. Towards a more sustainable taxation policy}

In [55] a more moderate penalty factors choice was advocated:

$$
\begin{aligned}
& q_{\sigma}=1, \quad w \leq 2, \\
& q_{\sigma}=c 4^{w-2}, \quad w>2,
\end{aligned}
$$

where $c$ is an order 1 constant. The authors called this choice "moderate," because sectional curvatures are not as big as in the draconian model. Big curvatures in general are not a desired feature of complexity geometry, because they are in tension with the desired properties of scrambling and switchback effect. The exponential behavior $q_{k} \propto 4^{k}$ in (4.10) is suggested by the draconian model: in such a case the behavior $q \propto 4^{n}$ of penalties is needed in order to have negative curvature. In this section we will consider some variations of this model, in which $q_{k} \propto \alpha^{k}$ for some appropriate constant $\alpha$.

The draconian model resembles a flat tax: all the weights bigger than 2 are treated the same. The middle-class exponents with $w \approx 3$ and the billionaires with $w \approx n$ pay exactly the same amount of taxes. The penalty choice in Eq. (4.10) goes in the direction of a more progressive taxation, because high incomes are taxed progressively. Still there is a minor source of inequality in Eq. (4.10): the very low income guys at $w=1$ are taxed just the same as the working class at $w=2$. In order to promote social justice we are motivated to introduce the following choice of penalties (see also [70])

$$
q_{\sigma}=\alpha^{w-1},
$$

which we will call "progressive" penalties. The scaling as $4^{k}$ at large $k$ is generalized as $\alpha^{k}$.

The model (4.11) simplifies in the large $\alpha$ limit, which can be used as an expansion parameter for the analytical understanding of the model. In particular, from Eq. (2.17) we can see that at large $\alpha$ sectional curvatures scale at most as $\alpha^{0}$. With the choice in Eq. (4.11), we expect that by construction the maximal complexity becomes infinity at fixed $n$ in the limit $\alpha \rightarrow \infty$, because one qubit operators cannot produce the most general operators in the unitary space. For example, they cannot produce unitaries that entangle two qubits that were previously unentangled. Physically, we will be interested in the limit of large but finite $\alpha$.

Moreover, we can consider generalizations of this basic model. In particular, we can generalize the choice in Eq. (4.11) as

$$
\begin{aligned}
& q_{\sigma}=1, \quad w \leq w_{0}, \\
& q_{\sigma}=\alpha^{w-w_{0}}, \quad w>w_{0},
\end{aligned}
$$

with $w_{0} \geq 2$. For $w_{0}=2$ and $\alpha=4$, we recover the model studied in [55]. With this choice of penalties, we expect that the maximal complexity at fixed $n$ does not diverge for $\alpha \rightarrow \infty$, because the combination of 1 and 2 qubits operators is universal and can be used to build an arbitrary operator in the unitary space. From Eq. (2.17), we can see that this model has the property that at large $\alpha$ sectional curvatures scale at most as $\alpha^{w_{0}-1}$. Therefore, the large $\alpha$ limit provides a singular geometry, as the curvature diverges.

\section{Progressive penalties}

We computed the curvatures as a power expansion in $\alpha$, at the leading order $\alpha^{0}$ and at next to leading order $\alpha^{-1}$. The cumbersome calculations are deferred to Appendix B.

At the leading order in $\alpha$, the scalar curvature is

$$
R=3 n\left(4^{n}-27^{n-1}\right) .
$$



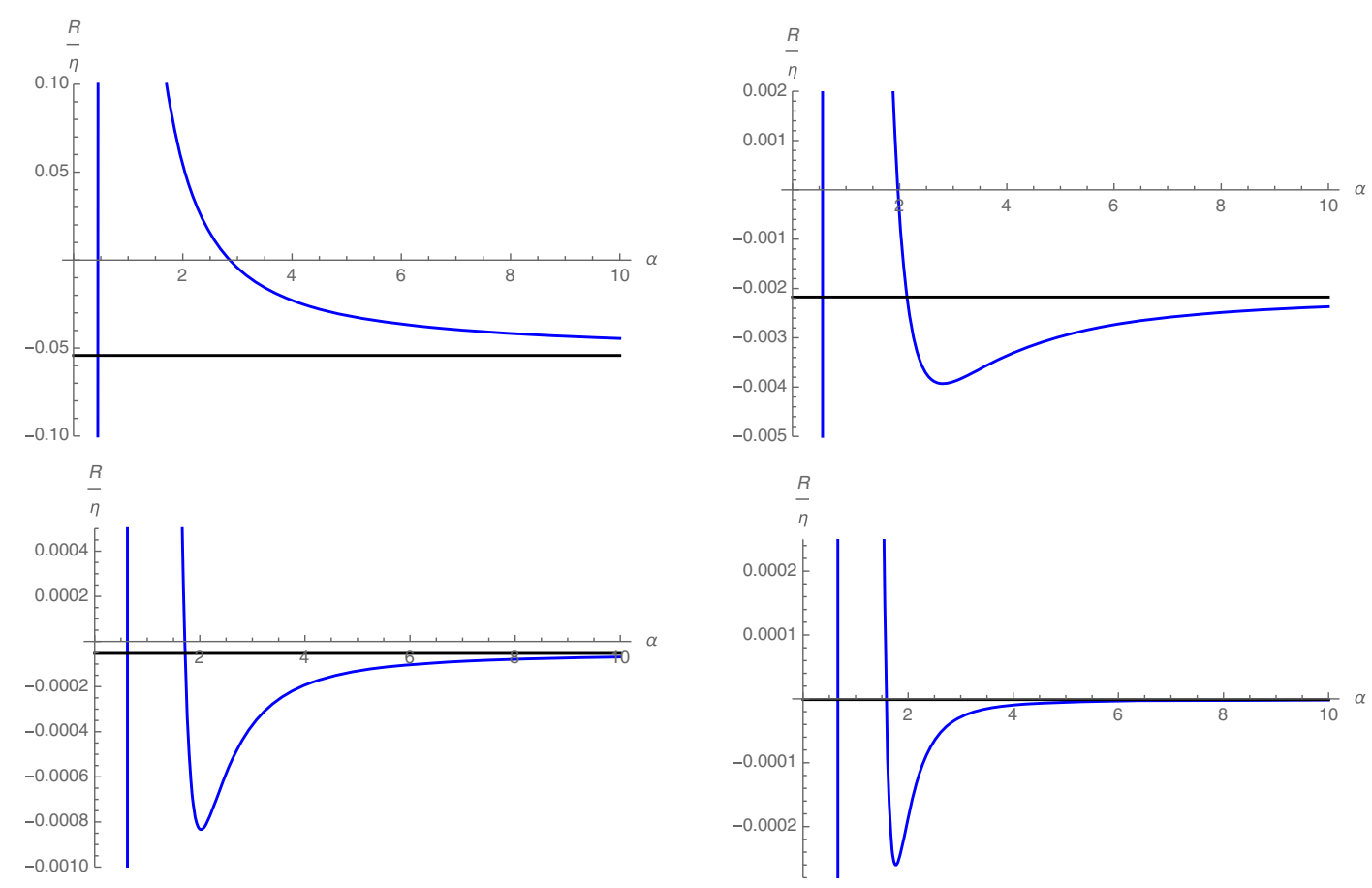

FIG. 2. The exact value of $R / \eta$ plotted as a function of $\alpha$ in the case of progressive penalties, for $n=5,10,15,20$. The asymptotic value at $\alpha \rightarrow \infty$ is shown in black. The minimum in the picture appears for $n \geq 8$. Increasing $n$, the shape of the minimum tends to become more and more steep and it is located at a lower value of $\alpha$. Note that when $n=20$, for values $\alpha \geq 4$ the result of the average sectional curvature at $\mathcal{O}\left(\alpha^{0}\right)$ is already very close to the exact result.

It is negative for $n \geq 3$ and comes just from two values of the sectional curvatures: $K=1$ with multiplicity $\mathcal{N}_{+}$and $K=-3$ with multiplicity $\mathcal{N}_{-}$, where

$$
\begin{aligned}
& \mathcal{N}_{+}=127^{n-1} n-32^{2 n+1} n+18 n \\
& \mathcal{N}_{-}=\frac{\mathcal{N}_{+}}{2}-3 n
\end{aligned}
$$

At next to leading order, the correction to the curvature is

$$
\delta R=\frac{9}{2} n(n-1) \frac{4^{n}}{\alpha} .
$$

In order to get a feeling on the average sectional curvature, it is convenient to divide $R$ by the total number of sectional curvatures between couples of elements in the basis, which we denote by

$$
\eta=\left(4^{n}-1\right)^{2}-\left(4^{n}-1\right) .
$$

The average sectional curvature becomes tiny at large $n$ and $\alpha$, i.e.,

$$
\bar{K}=\frac{R}{\eta} \approx-\frac{6}{7} n\left(\frac{7}{16}\right)^{n}+\frac{1}{\alpha} \frac{9}{4^{n}} \frac{n(n-1)}{2} .
$$

We do not have an analytic expression at higher order in $\alpha$ for the generic $n$ qubits case. However, if $n$ is fixed to be some not too large value, we can compute the exact result at all orders explicitly since the sum over the penalties contains a finite number of terms.

The exact value of the average sectional curvature as a function of $\alpha$ for a few values of $n$ is plotted in Fig. 2 . Nothing special happens for the value $\alpha=4$, which instead plays an important role for the draconian model. It is interesting that there is a minimum at finite $\alpha$. It turns out that the series expansion in $\alpha^{-1}$ for $\bar{K}$ is, at large $n$, an alternate sign series with slow rate of convergence. For example, in order to get the minimum in the plot for $\bar{K}$ when $n=10$, we have to expand up to the order $\alpha^{-5}$.

This choice of penalties for $\alpha \rightarrow \infty$ has many similarities with the one qubit case in Eq. (3.7), where $P=\beta Q \rightarrow \infty$ with $\beta$ constant and different from 1 . In both limits we expect that the maximal complexity diverges, and the sectional curvatures do not. Also, $R$ approaches a negative constant in both cases.

\section{STATE COMPLEXITY AND SUBMERSIONS}

Up to now, we have focused the discussion on the complexity of unitaries. In this section, we bring the attention of our reader to the geometry of the space of states. Geometrically, this space is naturally associated with a quotient of the space of unitaries where all the different unitary transformations that, starting from a given reference state, build the same state (up to a phase) are identified. 
The complexity of the state built in this way is then defined to be the minimum of the complexities of all the identified unitaries. Requiring that the state complexity is also obtained as a length on the space of states defines a map between two Riemannian manifolds, which turns out to be a Riemannian submersion. We recall its definition in Sec. VA, and we proceed in the subsequent sections in exploiting known results for Riemannian submersions.

In particular, O'Neill's formula relates the curvature of the space of states to the one of the space of unitaries, providing a lower bound on the curvature on states. This underlying geometrical structure allows a direct comparison of some class of geodesics, which we explore in Secs. V D and VI.

\section{A. Submersions}

For convenience of the reader, in this section we briefly review the concept of Riemannian sumbersions, referring to the textbooks $[65,71]$ for more details.

Let us consider two Riemannian manifolds $\left(M, g_{\alpha \beta}\right)$ with dimension $m$ and $\left(B, h_{\alpha \beta}\right)$ with dimension $b<m$ and a smooth map $\pi: M \rightarrow B$ with surjective differential $d \pi . d \pi$ is a map $d \pi: T M \rightarrow T B$, that for any $y \in M$ induces a linear map between the vector spaces $T_{y} M$ and $T_{x} B$, where $x=\pi(y)$. This map has maximal rank, and thus a kernel of dimension $f=m-b$. We will call $\mathcal{V}_{y}=\operatorname{ker}\left(d \pi_{y}\right)$ the vertical space at $y$. Its orthogonal complement in $T_{y} M$, induced by the metric $g$, is called the horizontal space at $y$ and denoted by $\mathcal{H}_{y}$. For the submersion to be Riemannian, $\mathcal{H}_{y}$ has to be identified with $T_{x} B$ in an isometric way, in other words

$$
g(X, Y)=h(d \pi(X), d \pi(Y)), \quad \forall X, Y \in \mathcal{H}_{y} .
$$

A pictorial depiction is shown in Fig. 3.

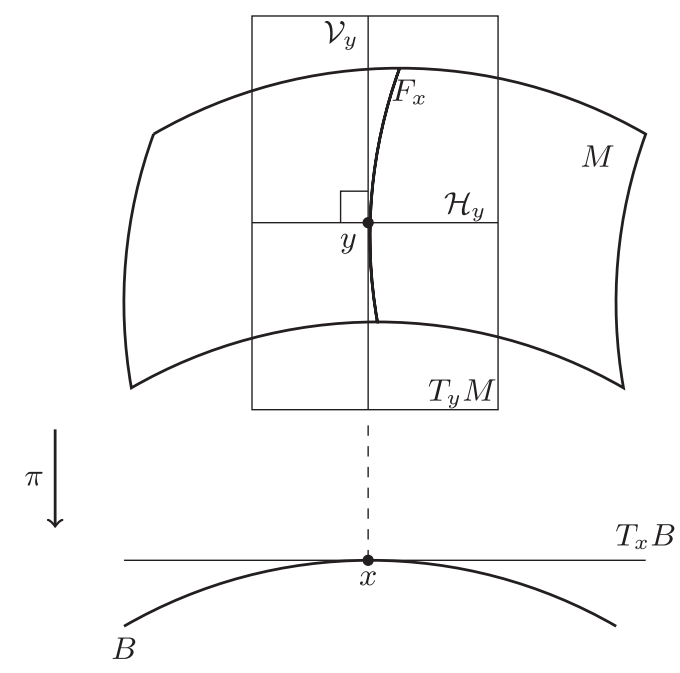

FIG. 3. A reproduction of a depiction of a submersion from [71].
Quotients of manifolds by an isometric group action provide interesting examples of submersion (see for example the textbooks [65,71]). Let $M$ be a Riemannian manifold and $G$ be a closed subgroup of the isometry group of $M$, and denote by $\pi$ the projection from $M$ to the quotient space $B=M / G$. This defines a natural metric on $B$ such that $\pi$ is a Riemannian submersion [65].

In the following sections, we make use of this construction to understand properties of the space of states from the complexity of unitaries.

\section{B. Submersions and complexity geometry}

Let us apply the notion of submersion to the complexity geometry. We take $M=S U\left(2^{n}\right)$ with a right-invariant metric (the unitary space) and $G$ as the subgroup of the isometries of $M$ which leaves the reference state invariant up to a phase. More precisely, we consider a unitary $U$ which generates the state $|\psi\rangle$ starting from the reference state $\left|\psi_{0}\right\rangle$

$$
U\left|\psi_{0}\right\rangle=|\psi\rangle
$$

We call unbroken subgroup the subgroup of $S U\left(2^{n}\right)$ that fixes the reference state up to a phase

$$
V\left|\psi_{0}\right\rangle=e^{i \phi}\left|\psi_{0}\right\rangle .
$$

Such a $V$ is an element of $S U\left(2^{n}-1\right) \times U(1)$. Thus, up to a phase, both $U^{\prime} \equiv U V$ and $U$ prepare the same state $|\psi\rangle$ :

$$
U^{\prime}\left|\psi_{0}\right\rangle=e^{i \phi}|\psi\rangle, \quad \Rightarrow \quad U^{\prime} \sim U
$$

Therefore we have a map from the unitary space to the quotient $B$ defined as

$$
\pi: \quad S U\left(2^{n}\right) \rightarrow B
$$

where

$$
B=\mathbb{C P} \mathbb{2}^{2^{n}-1}=\frac{S U\left(2^{n}\right)}{S U\left(2^{n}-1\right) \times U(1)} .
$$

This map is an isometric submersion, as we are going to prove writing it explicitly in a specific coordinate system.

In order to make contact with Sec. II, we take a diagonal penalty matrix in the basis of the generalized Pauli matrices, see Eq. (2.4), with the property

$$
\left\langle\sigma_{r}, \sigma_{s}\right\rangle=q_{r} \delta_{r s}=q_{r} \frac{1}{2^{n}} \operatorname{Tr}\left(\sigma_{r} \sigma_{s}\right)
$$

For the states metric it is more convenient to do a change of basis. We can identify a basis for broken generators $\rho_{k}$ and unbroken ones $\tau_{a}$ : 


$$
\begin{gathered}
\omega_{l}=\left(\rho_{k}, \tau_{a}\right), \quad 1 \leq k \leq 2(K-1), \\
1 \leq a \leq(K-1)^{2}, \quad K=2^{n},
\end{gathered}
$$

with normalization

$$
\operatorname{Tr}\left(\omega_{l} \omega_{m}\right)=\delta_{m l}
$$

We can express

$$
\begin{aligned}
\sigma_{r} & =\sum_{l} \omega_{l} \operatorname{Tr}\left(\omega_{l} \sigma_{r}\right), \\
\omega_{l} & =\frac{1}{2^{n}} \sum_{r} \sigma_{r} \operatorname{Tr}\left(\omega_{l} \sigma_{r}\right) .
\end{aligned}
$$

Then we can find the penalty scalar product in the basis $\omega^{k}$ :

$M_{l m}=\left\langle\omega_{l}, \omega_{m}\right\rangle=\frac{1}{2^{2 n}} \sum_{r} q_{r} \operatorname{Tr}\left(\omega_{l} \sigma_{r}\right) \operatorname{Tr}\left(\omega_{m} \sigma_{r}\right)$.

This discussion also applies to the case where $M_{l m}$ is a generic symmetric matrix. Let us introduce the following notation for the exponential of broken and unbroken generators

$$
U_{\theta}=e^{i \theta_{k} \rho_{k}}, \quad V_{\lambda}=e^{i \lambda_{a} \tau_{a}},
$$

where the variables $\theta_{k}$ denote the coordinates in the state space and $\lambda_{a}$ are the additional coordinates that define the unitary space. A generic element of $S U(K)$ can be written as $U=U_{\theta} V_{\lambda}$. Then we can compute

$$
\begin{aligned}
d U U^{\dagger} & =\left(d U_{\theta} V_{\lambda}+U_{\theta} d V_{\lambda}\right) V_{\lambda}^{\dagger} U_{\theta}^{\dagger}, \\
& =d U_{\theta} U_{\theta}^{\dagger}+U_{\theta} d V_{\lambda} V_{\lambda}^{\dagger} U_{\theta}^{\dagger},
\end{aligned}
$$

where

$$
d U_{\theta}=\frac{\partial U_{\theta}}{\partial \theta_{k}} d \theta_{k}, \quad d V_{\lambda}=\frac{\partial V_{\lambda}}{\partial \lambda_{j}} d \lambda_{j}
$$

In this way the right-invariant forms defined on $S U(K)$ are given by

$$
\begin{aligned}
X_{r} & =-i \operatorname{Tr}\left(d U U^{\dagger} \omega_{r}\right), \\
& =-i\left(\operatorname{Ad}_{U_{\theta}^{\dagger}}\right)_{r s} \operatorname{Tr}\left\{\left(U_{\theta}^{\dagger} d U_{\theta}+d V_{\lambda} V_{\lambda}^{\dagger}\right) \omega_{s}\right\},
\end{aligned}
$$

where we have used the adjoint action

$$
U_{\theta}^{\dagger} \omega_{r} U_{\theta}=\left(\operatorname{Ad}_{U_{\theta}^{\dagger}}\right)_{r s} \omega_{s} .
$$

We can now write the metric in the unitary space as

$$
d s^{2}=M_{r s} X_{r} X_{s}=\tilde{M}_{l m}\left(u_{l}+v_{l}\right)\left(u_{m}+v_{m}\right),
$$

where

$$
\begin{aligned}
\tilde{M}_{l m} & =M_{r s}\left(\operatorname{Ad}_{U_{\theta}^{\dagger}}\right)_{r l}\left(\operatorname{Ad}_{U_{\theta}^{\dagger}}\right)_{s m}, \\
u_{s} & =-i \operatorname{Tr}\left\{U_{\theta}^{\dagger} d U_{\theta} \omega_{s}\right\}, \\
v_{s} & =-i \operatorname{Tr}\left\{d V_{\lambda} V_{\lambda}^{\dagger} \omega_{s}\right\},
\end{aligned}
$$

in such a way that $\tilde{M}_{l m}$ depends just on $\theta_{k}, u_{s}$ contains just $\left(\theta_{k}, d \theta_{k}\right)$ and $v_{s}$ contains just $\left(\lambda_{a}, d \lambda_{a}\right)$.

Now it is convenient to split the indices in $\omega_{r}$ in indices corresponding to broken and unbroken generators, as in Eq. (5.8). We have that $v_{i}=0$ for $i$ corresponding to a broken index. Then we can write the unitary metric Eq. (5.17) as

$$
\begin{aligned}
d s^{2} & =\left(\begin{array}{ll}
u_{i} & u_{a}+v_{a}
\end{array}\right)\left(\begin{array}{cc}
\tilde{M}_{i j} & \tilde{M}_{i b} \\
\tilde{M}_{a j} & \tilde{M}_{a b}
\end{array}\right)\left(\begin{array}{c}
u_{j} \\
u_{b}+v_{b}
\end{array}\right), \\
& =\left(\tilde{M}_{i j}-\tilde{M}_{i c} \tilde{M}_{c a}^{-1} \tilde{M}_{a j}\right) u_{i} u_{j}+\tilde{M}_{a b} f_{a} f_{b},
\end{aligned}
$$

where we introduced

$$
f_{a}=v_{a}+u_{a}+\tilde{M}_{a d}^{-1} \tilde{M}_{d j} u_{j}
$$

The problem of finding the minimal infinitesimal operator which synthesizes the state of coordinates $\theta_{k}+d \theta_{k}$ from the state with coordinates $\theta_{k}$ is then solved by the equation $f_{a}=0$, because the term $\tilde{M}_{a b} f_{a} f_{b}$ in Eq. (5.19) is positive definite. This construction generalizes the result in [60] to arbitrary number of qubits.

We can then identify the metric on the state space $B$ as

$$
d s_{S}^{2}=\left(\tilde{M}_{i j}-\tilde{M}_{i c} \tilde{M}_{c a}^{-1} \tilde{M}_{a j}\right) u_{i} u_{j} .
$$

We explicitly checked that the metric in the space of states $\mathbb{C P} P^{1}$ for a single qubit coincides with the result found in [60]. In Appendix $C$ we will see how to apply this result to qutrits.

From Eq. (5.19), it follows that the projection map $\pi$ from $M$ to $B$

$$
\pi:\left(\theta_{k}, \lambda_{j}\right) \mapsto\left(\theta_{k}\right)
$$

is a Riemannian submersion, where $\pi^{-1}\left(\theta_{k}\right)$ is parametrized by $\lambda_{k}$, for fixed $\theta_{k}$. The explicit expression for the horizontal spaces at arbitrary $\theta_{k}$ is given by $f_{a}(X)=0$ for any generic vector $X$ in the tangent space.

\section{Submersions and curvature}

We can use O'Neill's formula [61] to relate the sectional curvatures of states $K_{S}$ to the ones of unitaries $K$ :

$K_{S}\left(\tilde{h}_{1}, \tilde{h}_{2}\right)=K\left(h_{1}, h_{2}\right)+\frac{3}{4} \frac{\left|\mathcal{V}\left(\left[h_{1}, h_{2}\right]\right)\right|^{2}}{\left|h_{1}\right|^{2}\left|h_{2}\right|^{2}-\left\langle h_{1}, h_{2}\right\rangle^{2}}$, 
where $\mathcal{V}$ is the projector on the vertical subspace, $\langle\ldots\rangle$ is the scalar product from the metric of the manifold $M,|\ldots|$ is the norm induced by the scalar product, $\tilde{h}_{k}=d \pi\left(h_{k}\right)$ are vectors fields in the state space, $h_{k}$ are horizontal fields in the unitary space, $\left[h_{1}, h_{2}\right]$ is the commutator of the vector fields in the unitary space.

This expression shows that the sectional curvature of a plane in the space of states can be always expressed as sectional curvature of an appropriate plane in the unitary space plus a positive definite contribution coming from the commutator of horizontal vectors. It can be used to compute the curvatures in the state space without even knowing its metric. As an illustrative example, we apply Eq. (5.22) to the one qubit case in Appendix D.

\section{Submersions and geodesics}

The relation between geodesics in $B$ and geodesics in $M$ for generic submersions was studied in [62]. An important result is that if a geodesic in $M$ is horizontal at some point, it remains horizontal. Then the projection by $\pi$ of an horizontal geodesic is a geodesic in the space of states $B$. As a general result, we have that for submersions from complete manifolds $M$ as our unitary space, every geodesic of $B$ can be built as the projection of a horizontal geodesic in $M$. It is important to stress that the projection of a geodesic that is not horizontal in general does not provide a geodesic on $B$.

We know from Eq. (2.9) that the exponential of an eigenvector of the penalty matrix $\mathcal{G}$ is a geodesic in the unitary space. Combining with the previous result, the exponential of an eigenstate of $\mathcal{G}$ which is also perpendicular to the unbroken subgroup at the origin, gives a geodesic in the state space $B$. This property provides us a simple class of geodesics in some particular situations. In the 1-qubit case, this is studied in Sec. VIC.

Let us instead consider the 2-qubits case with penalties depending just on the weights. Taking as reference state $|00\rangle$, the unbroken subgroup is generated by the following generators:

$$
\begin{aligned}
& \mathbb{1} \otimes \sigma_{z}, \quad \sigma_{z} \otimes \mathbb{1}, \quad \sigma_{z} \otimes \sigma_{z}, \\
& \sigma_{x} \otimes\left(\mathbb{1}-\sigma_{z}\right), \quad \sigma_{y} \otimes\left(\mathbb{1}-\sigma_{z}\right), \\
& \left(\mathbb{1}-\sigma_{z}\right) \otimes \sigma_{x}, \quad\left(\mathbb{1}-\sigma_{z}\right) \otimes \sigma_{y}, \\
& \sigma_{x} \otimes \sigma_{y}-\sigma_{y} \otimes \sigma_{x}, \\
& \sigma_{x} \otimes \sigma_{x}+\sigma_{y} \otimes \sigma_{y} .
\end{aligned}
$$

The orthogonal complement to this space is generated by

$$
\begin{aligned}
& \sigma_{x} \otimes\left(\mathbb{1}+\alpha \sigma_{z}\right), \quad \sigma_{y} \otimes\left(\mathbb{1}+\alpha \sigma_{z}\right), \\
& \left(\mathbb{1}+\alpha \sigma_{z}\right) \otimes \sigma_{x}, \quad\left(\mathbb{1}+\alpha \sigma_{z}\right) \otimes \sigma_{y}, \\
& S_{2}^{-}=\sigma_{x} \otimes \sigma_{y}+\sigma_{y} \otimes \sigma_{x}, \\
& S_{2}^{+}=\sigma_{x} \otimes \sigma_{x}-\sigma_{y} \otimes \sigma_{y},
\end{aligned}
$$

where $\alpha$ is a coefficient ${ }^{2}$ dependent on the penalty factors, chosen to ensure orthogonality with unbroken generators in Eq. (5.24). Note that just the last two generators $S_{2}^{ \pm}$in Eq. (5.25) have a definite weight $w=2$, and so just these two operators generate exponential horizontal geodesics.

We can generalize this arguments to $n$ qubits as follows. Let us take as reference state $|00 \ldots 0\rangle$. Let us consider the action of a infinitesimal transformation on this state, with $w=n$ and which contains just $\sigma_{x}$ and $\sigma_{y}$ entries in the tensor product. This operator will rotate the state as

$$
|00 \ldots 0\rangle \rightarrow|00 \ldots 0\rangle+\epsilon|11 \ldots 1\rangle,
$$

where $\epsilon$ is an infinitesimal complex number. This sector of operators contain $2^{n}$ generators; out of this set, a vector space of dimension $2^{n}-2$ operators is unbroken. So, in the $w=n$ sector which contain just tensor products of $\sigma_{x}$ and $\sigma_{y}$ we can always find a broken dimension 2 subspace which is orthogonal to the vertical space.

Let us build these generators explicitly. We introduce

$$
A_{s}^{n}=\frac{1}{\left(\begin{array}{c}
n \\
s
\end{array}\right)} \sum_{\left(k_{1}, \ldots, k_{n}\right)} \sigma_{k_{1}} \otimes \ldots \otimes \sigma_{k_{n}}
$$

where the sum runs over all the permutations $\left(k_{1}, \ldots, k_{n}\right)$ which contain $s$ generators $\sigma_{y}$ and $n-s$ generators $\sigma_{x}$. Then the two generators

$$
S_{n}^{+}=\sum_{0 \leq k \leq n}^{k \text { even }} i^{k} A_{k}^{n}, \quad S_{n}^{-}=\sum_{0 \leq k \leq n}^{k \text { odd }} i^{k+1} A_{k}^{n},
$$

are both broken by the reference state and orthogonal to all the unbroken $w=n$ generalized Pauli matrices which contain just $\sigma_{x}$ and $\sigma_{y}$ in the tensor product. This construction generalizes to $n$ qubits the two operators in the last line of Eq. (5.25).

Then we can look for other generators orthogonal to the vertical space. We can consider a generalized Pauli matrix of the form $S_{n-1}^{ \pm} \otimes\left(\mathbb{1}+\alpha_{1} \sigma_{z}\right)$ with the coefficient chosen in such a way that it is orthogonal to $S_{n-1}^{ \pm} \otimes\left(\mathbb{1}-\sigma_{z}\right)$. This involves a linear combination of weight $n$ and $n-1$ generators and in general one can find $2\left(\begin{array}{l}n \\ 1\end{array}\right)$ such operators. One can iterate the construction, looking for generators of the form

$$
S_{n-s}^{ \pm} \otimes\left(\mathbb{1}+\alpha_{s} \sigma_{z}\right)^{s},
$$

and determine $\alpha_{s}$ in such a way that (5.29) is orthogonal to the unbroken operators

\footnotetext{
${ }^{2}$ The precise value is completely irrelevant for the following discussion.
} 


$$
S_{n-s}^{ \pm} \otimes\left(\mathbb{1}-\sigma_{z}\right) \otimes 1^{b} \otimes \sigma_{z}^{c}
$$

where $b, c$ are some integer numbers. For each integer $s$, the operators in (5.29) are linear combinations of weight $w$ generators with

$$
n-s \leq w \leq n .
$$

There are $2\left(\begin{array}{l}n \\ s\end{array}\right)$ of such operators, with $1 \leq s \leq n$. In this way one can build all the $2^{n}-1$ horizontal vectors in the unitary space, which project to the $\mathbb{C} \mathbb{P}^{2^{n}-1}$ directions in the state space. A broken unitary labelled by $s$ is a linear combination of generalized Pauli matrices with weight $w$ with $n-s \leq w \leq n$.

If the penalties of each weight $q_{w}$ are all different (as in the progressive model), just the $s=0$ broken unitaries $S_{n}^{ \pm}$ are penalty eigenstates. This is the most generic case. The only exponential horizontal geodesics are generated by linear combinations of $S_{n}^{+}$and $S_{n}^{-}$.

If some penalties for different weights are degenerate, we can find more eigenstates of the penalties which are orthogonal to the unbroken subgroup. For example, in the draconian model all the weights with $3 \leq w \leq n$ are equally penalized, so all the broken unitaries with $0 \leq s \leq$ $n-3$ generate projectable exponential geodesics.

There is a relation between conjugate points in $M$ and $B$ [62]. Let us consider a horizontal geodesic

$$
\gamma(t):[a, b] \rightarrow M
$$

and let $\gamma\left(t_{0}\right)$ be the first conjugate point of $\gamma$ along the geodesic starting form $\gamma(a)$. Then the projected geodesic $\beta(t)=\pi(\gamma(t))$ has a conjugate point for $t_{0}^{\prime} \leq t_{0}$.

\section{TOWARDS AN EXPONENTIAL COMPLEXITY}

The definitions of unitary and state complexity require the minimization of the length of a path connecting the identity with a generic unitary, or the reference state to the target state, respectively. In the following, we exploit the techniques developed in the previous sections to find explicit classes of geodesics and to find their conjugate points, which play an important role in the minimization process.

\section{A. Conjugate points and Raychaudhury equation}

An important problem in the geometric approach to complexity is to determine the minimal length geodesics that connect the identity to a given unitary. From a general result in Riemannian geometry, a geodesic does not minimize lengths anymore after its first conjugate point. This is not a necessary condition: there could be a globally shorter path before the first conjugate point.

A useful tool to study conjugate points is the Raychaudhury equation (see, e.g., [72] for a review). Let us consider a congruence of geodesics which is orthogonal to a family of hypersurfaces in an arbitrary Riemannian manifold. Let us denote by $u^{\alpha}$ the tangent vector field to the geodesics, with $u^{\alpha} u_{\alpha}=1$. The geodesics are in affine parametrization, i.e., $u^{\beta} D_{\beta} u^{\alpha}=0$, where $D_{\beta}$ is the covariant derivative. The deviation vectors $\xi^{\mu}$ are taken orthogonal to $u^{\alpha}$, i.e., $\xi^{\alpha} u_{\alpha}=0$. We can define the transverse part of the metric as

$$
h_{\alpha \beta}=g_{\alpha \beta}-u_{\alpha} u_{\beta},
$$

and the tensor

$$
B_{\alpha \beta}=D_{\beta} u_{\alpha}
$$

which can be shown to be symmetric if the congruence of geodesics is orthogonal to a family of hypersurfaces. Morever $B_{\alpha \beta}$ can be decomposed in the trace and traceless part

$$
B_{\alpha \beta}=\frac{1}{d-1} \Theta h_{\alpha \beta}+\sigma_{\alpha \beta},
$$

where $d$ is the dimension of space, $\Theta$ is the expansion scalar and $\sigma_{\alpha \beta}$ the (traceless and symmetric) shear tensor. The expansion scalar $\Theta$ measures the time derivative of an infinitesimal transverse volume $\Delta V$ of the geodesic, i.e.,

$$
\Theta=\frac{1}{\Delta V} \frac{d \Delta V}{d \lambda} .
$$

If the scalar $\Theta$ approaches $-\infty$ in some point $r$ along a geodesic, it detects the presence of conjugate points for our congruence of geodesics. This means that the geodesic that we are studying does not anymore give us the minimal distance for points beyond $r$. The Raychaudhury equation determines the evolution of $\Theta$ along the geodesic flow:

$$
\frac{d \Theta}{d \lambda}=-\frac{1}{d-1} \Theta^{2}-\sigma^{\alpha \beta} \sigma_{\alpha \beta}-R_{\alpha \beta} u^{\alpha} u^{\beta},
$$

where $R_{\alpha \beta}$ is the Ricci tensor and $\lambda$ is an affine parameter. There exists also an equation for the traceless part $\sigma_{\alpha \beta}$, see, e.g., [73]. We discuss this equation in Appendix E.

\section{B. An application to a simple class of geodesics}

From Eq. (2.9), we know that, in the unitary space, the exponential of eigenvectors of the penalty factors matrix $\mathcal{G}$ gives us a class of geodesics, which we call "exponential geodesics." It is particularly convenient to apply the Raychaudhury equation to this class of geodesics, which have constant $R_{\alpha \beta} u^{\alpha} u^{\beta}$. If we neglect the term $\sigma^{\alpha \beta} \sigma_{\alpha \beta}$ in Eq. (6.5), it can be solved analytically. In general this term is nonzero (see Appendix E), but it is positive definite. 
So, neglecting the $\sigma^{\alpha \beta} \sigma_{\alpha \beta}$ term gives us an upper bound for the presence of a conjugate point along a geodesic.

Let us first solve Eq. (6.5) in the limit $\Theta \rightarrow \infty$, as it is the case for a family of geodesics starting from the same point. In this case we can neglect $R_{\alpha \beta} u^{\alpha} u^{\beta}$, leading to

$$
\dot{\Theta}+\frac{1}{d-1} \Theta^{2}=0, \quad \Theta=\frac{d-1}{\lambda-k},
$$

where $k$ is an integration constant. This approximation is the same as considering the flat space limit. In order to consider a family of geodesics that start at the same point at $\lambda=0$, we set $k=0$. Let us now consider

$$
\dot{\Theta}+\frac{1}{d-1} \Theta^{2}+B=0,
$$

where $B=R_{\alpha \beta} u^{\alpha} u^{\beta}$. The conjugate point, in this approximation, shows up only for $B>0$. Requiring that at small $\lambda$ the solution reproduces the flat space one $\Theta=(d-1) / \lambda$, we find

$$
\Theta=\sqrt{B(d-1)} \cot \left(\sqrt{\frac{B}{d-1}} \lambda\right),
$$

and so it has a conjugate point at

$$
\lambda_{0}=\frac{\pi \sqrt{d-1}}{\sqrt{B}} .
$$

Since $\sigma^{\alpha \beta} \sigma_{\alpha \beta}$ is a positive-definite quantity, the value of $\lambda_{0}$ provides an upper bound for the distance $\lambda_{c}$ of the conjugate point from the origin:

$$
\lambda_{c} \leq \lambda_{0}=\frac{\pi \sqrt{d-1}}{\sqrt{R_{\alpha \beta} u^{\alpha} u^{b}}} .
$$

Note that, keeping the Ricci curvature fixed, $\lambda_{0}$ scales exponentially with the number of qubits due to the factor $\sqrt{d-1} \approx 2^{n}$. This is a first evidence of the exponential nature of the maximal complexity.

\section{One qubit}

In order to make the discussion concrete with a clear example we will consider the one qubit case, see Sec. III A. In this case the unitary manifold is a generalized Berger sphere and an explicit expression for the metric is available. Introducing the coordinates $\left(\theta_{x}, \theta_{y}, \theta_{z}\right)$ to parametrize the unitary

$$
U=e^{i \sigma_{z} \theta_{z}} e^{i \sigma_{y} \theta_{y}} e^{i \sigma_{x} \theta_{x}},
$$

the metric can be written explicitly:

$$
g_{i j}=\frac{1}{2}\left(\begin{array}{ccc}
\Xi & \Psi & 2 P \sin 2 \theta_{y} \\
\Psi & \Sigma & 0 \\
2 P \sin 2 \theta_{y} & 0 & 2 P
\end{array}\right),
$$

where

$$
\begin{aligned}
& \Xi=2\left(P \sin ^{2} 2 \theta_{y}+\cos ^{2} 2 \theta_{y}\left(Q \sin ^{2} 2 \theta_{z}+\cos ^{2} 2 \theta_{z}\right)\right), \\
& \Psi=(1-Q) \cos 2 \theta_{y} \sin 4 \theta_{z}, \\
& \Sigma=(Q-1) \cos 4 \theta_{z}+Q+1
\end{aligned}
$$

We know from the general analysis that the exponentials of $\sigma_{x}, \sigma_{y}, \sigma_{z}$ are geodesics, with

$$
\begin{array}{lll}
G_{x}: & \theta_{x}=\lambda, & \theta_{y}=\theta_{z}=0, \\
G_{y}: & \theta_{y}=\frac{\lambda}{\sqrt{Q}}, & \theta_{x}=\theta_{z}=0, \\
G_{z}: & \theta_{z}=\frac{\lambda}{\sqrt{P}}, & \theta_{x}=\theta_{y}=0,
\end{array}
$$

as can be also checked directly from the geodesic equations of the metric (6.12).

We have seen that the presence of conjugate points on this simple class of geodesics can be detected by the Ricci tensor:

$$
\begin{aligned}
& R_{x}=\frac{2\left(1-(P-Q)^{2}\right)}{P Q}, \\
& R_{y}=\frac{2(Q+P-1)(Q-P+1)}{P Q}, \\
& R_{z}=\frac{2(P+Q-1)(P-Q+1)}{P Q},
\end{aligned}
$$

where we denote $R_{x, y, z} \equiv R_{\sigma_{x}, \sigma_{y}, \sigma_{z}}$.

Conjugate points of the geodesic $G_{k}$ in Eq. (6.14) occur in the regions of the parameter space $(P, Q)$ where the corresponding $R_{k}$ is positive, see Fig. 4 . In particular, each of the geodesics $G_{k}$ for $k=x, y, z$ develops a conjugate point in the region where $R_{k}>0$ for

$$
\lambda_{c} \leq \lambda_{0}, \quad \lambda_{0}=\frac{\pi \sqrt{2}}{\sqrt{R_{k}}} .
$$

A plot of an example of conjugate point is shown in Fig. 4 in stereographic projection.

Using Eq. (E1), it is also possible to include the $\sigma^{\alpha \beta} \sigma_{\alpha \beta}$ corrections in order to determine in general the exact location of the conjugate points. From such an equation, we can show that $\sigma^{\alpha \beta}$ vanishes for $G_{x}$ in the $P=Q$ case, for $G_{y}$ in the $P=1$ case and for $G_{z}$ in the $Q=1$ case (see Appendix E). We have then a few exact results:

(i) For $Q=1, G_{z}$ has a conjugate point at $\lambda=\frac{\pi}{\sqrt{P}}$.

(ii) For $P=1, G_{y}$ has a conjugate point at $\lambda=\frac{\pi}{\sqrt{Q}}$. 

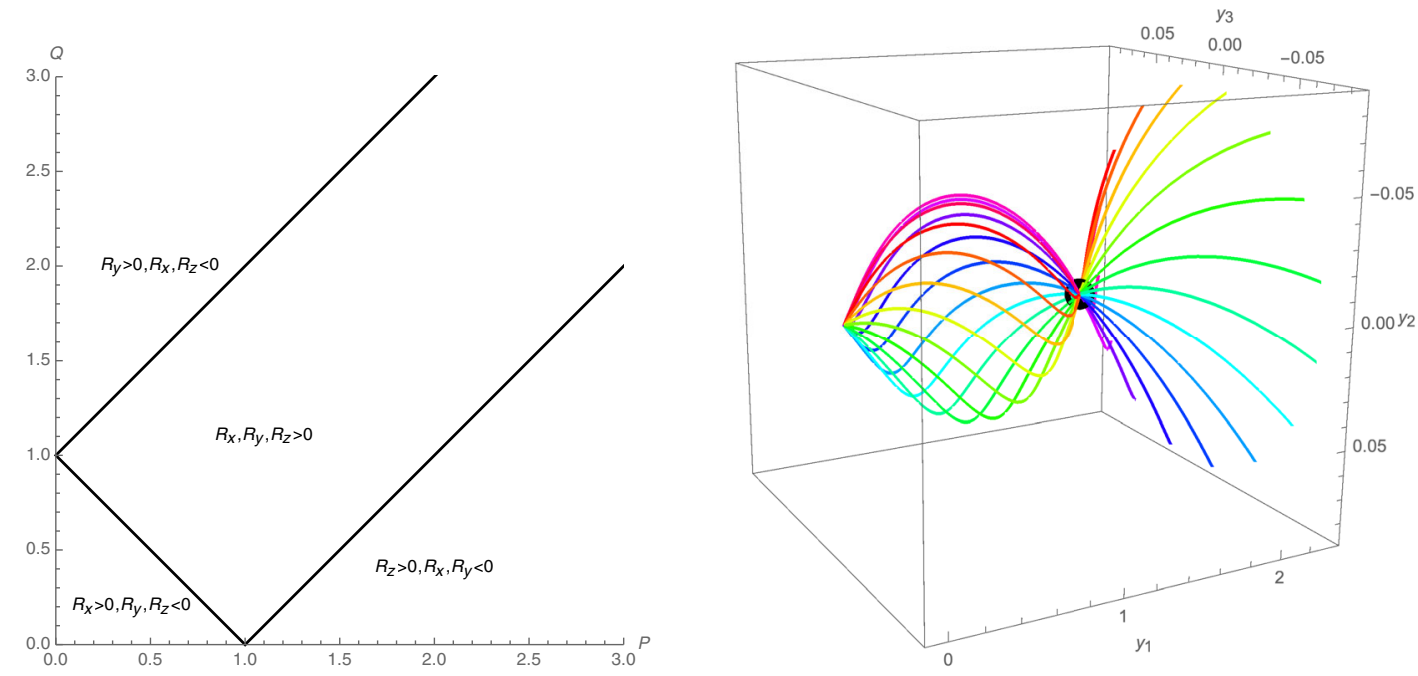

FIG. 4. Left: regions where each $R_{x, y, z}$ is positive. Right: example of an exact conjugate point (the black spot) of geodesics for $P=Q=0.4$ in stereographic projection.

(iii) For $P=Q, G_{x}$ has a conjugate point at $\lambda=\pi P$ (see the black spot in Fig. 4).

In particular, it is interesting to consider the limit in Eq. (3.3), with $P=1$ and $Q \rightarrow \infty$. In this case the only exponential geodesic with a conjugate point is $G_{y}$. In the limit $Q \rightarrow \infty$ the conjugate point moves very close to the origin, at $\theta_{y}=\pi / Q$ and at $\lambda=\pi / \sqrt{Q}$. The $G_{y}$ geodesic is then minimizing only very close to the origin, and the limit is singular. Indeed we already expected a singularity from the behavior of curvatures, see Eq. (3.4). Also, sending the penalty $Q$ to infinity does not correspond to getting a big complexity in the $\sigma_{y}$ direction: a shortcut with length scaling as $1 / \sqrt{Q}$ is for sure available just after the conjugate point. This is an indication of low maximal complexity and it is correlated to a singular limit in the curvature.

It is also interesting to consider the limit in Eq. (3.5), where $P=Q \rightarrow \infty$. The Ricci curvatures are all positive:

$$
R_{x}=\frac{2}{P^{2}}, \quad R_{y}=R_{z}=\frac{4}{P}-\frac{2}{P^{2}} .
$$

In this case $G_{x}$ has an exact conjugate point at $\theta_{x}=\lambda=$ $\pi P$, while $G_{y, z}$ have conjugate points for $\lambda \lesssim \pi \sqrt{P / 2}$, which correspond to $\theta_{y}, \theta_{z}$ of order 1 . There is no singularity in geodesic, as expected from the curvatures in (3.6). Note that, while the distance of the conjugate point in $G_{y, z}$ diverges, their position in the coordinate $\theta_{y, z}$ approaches a finite limit for $P \rightarrow \infty$. The limit of large penalty indeed may correspond to a large maximal complexity, because no obvious shortcuts are available. This is supported by numerical computations: the points with large complexity lay nearby the conjugate point, and so the maximal complexity scales as $\sqrt{P}$.

In the one qubit case, the exponential geodesics on unitary space can be projected to the states space using the submersion, as explained in Sec. V D. Taking as a reference state $|0\rangle$, the unbroken subgroup is generated by $\sigma_{z}$. The geodesics shot in the orthogonal directions $\sigma_{x}$ and $\sigma_{y}$ are then horizontal and projectable. For generic $P, Q$ there are then two exponential horizontal geodesics. The corresponding geodesics on states can be obtained by the projection of these curves by the submersion $\pi$.

It is more intuitive to plot the geodesics in the states space, since it is a two-dimensional space. In the one qubit case, the metric for states in the standard Bloch sphere coordinates $(\theta, \phi)$ is

$$
g_{i j}=\frac{1}{\Psi}\left(\begin{array}{ll}
\Lambda_{11} & \Lambda_{12} \\
\Lambda_{21} & \Lambda_{22}
\end{array}\right)
$$

where

$$
\begin{aligned}
\Lambda_{11} & =P \cos ^{2} \theta \cos ^{2} \phi+P Q \cos ^{2} \theta \sin ^{2} \phi+Q \sin ^{2} \theta, \\
\Lambda_{12} & =\Lambda_{21}=P(Q-1) \sin \theta \cos \theta \sin \phi \cos \phi, \\
\Lambda_{22} & =P \sin ^{2} \theta\left(Q \cos ^{2} \phi+\sin ^{2} \phi\right), \\
\Psi & =4\left\{\sin ^{2} \theta \sin ^{2} \phi+P \cos ^{2} \theta+Q \sin ^{2} \theta \cos ^{2} \phi\right\} .
\end{aligned}
$$

We checked numerically that the projection of the horizontal geodesics in the unitary space corresponds to geodesics in the states space, as is required by general results on submersions.

It is then interesting to plot the geodesics for the case of large $P$ and $Q$ in the state space. In Fig. 5 the geodesics for the case $P=Q=10$ on the Bloch sphere are shown. In particular, we see that the maximal complexity region lies just before the conjugate point in $\sigma_{y}$. Such a point lies inside the drop delimited by the self intersection of the black curve. As it is clear from the figure, no geodesics of length less than $\lambda$ can penetrate inside the drop. 


\section{Draconian model}

In order to study conjugate points in the draconian model we can use the results from [5] for $R_{\sigma}$, where $\sigma$ is a generalized Pauli matrix with weight $w$ :

$$
\begin{array}{ll}
w=1, & R_{\sigma}=2(3 n-2)+\frac{1}{q^{2}}\left(\frac{4^{n}}{2}-2(3 n-2)\right), \\
w=2, & R_{\sigma}=-24 q(n-2)+8(6 n-11)+\frac{1}{q^{2}}\left(\frac{4^{n}}{2}-8(3 n-5)\right), \\
w=3, & R_{\sigma}=\frac{1}{q}\left(12 q^{2}+\frac{4^{n}}{2}+36(n-3)-\frac{1}{q} 12(3 n-8)\right), \\
w \geq 4, & R_{\sigma}=\frac{1}{q}\left(\frac{4^{n}}{2}+4 w(3 n-2 w)-\frac{1}{q} 4 w(3 n-2 w)\right) .
\end{array}
$$

These expressions are valid for arbitrary $n$ and $q$. In particular, for $q=1$ we recover the cases with uniform penalties $q(w)=1$, where all the $R_{\sigma}$ are the same, i.e., $R_{\sigma}=4^{n} / 2$. In order to have negative scalar curvature, we have to scale $q$ with $n$ as $q \approx \mathcal{O}\left(4^{n}\right)$.

In studying conjugate points along the exponential geodesics, it is interesting to consider not only the distance $\lambda$ from the origin, but also their position in a coordinate $\theta$, which runs along the geodesic and does not scale with the penalty. We can define $\theta$ as the length in the case with all the penalties $q_{\sigma}=1$ (bi-invariant metric). In our normalization, an exponential geodesic can be described by

$$
U(\theta)=\exp \frac{i \theta \sigma}{\sqrt{2^{n}}},
$$

where $\sigma$ is the generalized Pauli matrix pointing in the direction of the given exponential geodesic. Note that this geodesic comes back to the identity matrix after a period

$$
\theta_{p}=\pi 2^{1+n / 2} .
$$

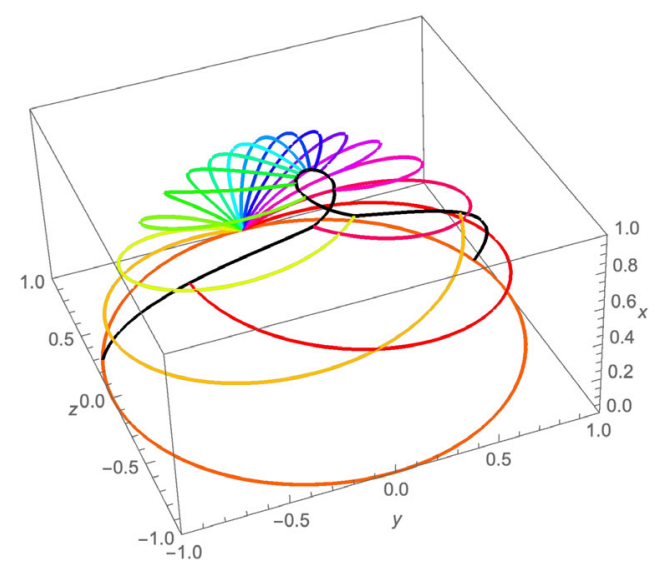

FIG. 5. Geodesics with length $\lambda=2.5$ for $Q=10, P=10$. The geodesics are plotted in different colors. The endpoints of the various curves are represented in black.
In the large $n$ limit of the unpenalized case $q(w)=1$, Eq. (6.10) gives that for all the weights $w$

$$
\lambda_{0}=\theta_{0} \approx \pi \sqrt{2}
$$

In every direction, the cut point must then be realized for $\lambda \leq \lambda_{0}$. This implies that the maximal complexity is less than $\pi \sqrt{2}$, which is independent of $n$.

Let us now consider the regime with negative scalar curvature $q \approx \mathcal{O}\left(4^{n}\right)$. We can use Eq. (6.10) with $d=$ $4^{n}-1$ to get an estimate of the distance of conjugate points from the origin:

$$
\begin{array}{ll}
w=1, & \lambda_{0} \approx \frac{\pi 2^{n}}{\sqrt{6 n}}, \quad \theta_{0}=\lambda_{0}, \\
w=3, \quad \lambda_{0} \approx \frac{\pi 2^{n}}{\sqrt{12 q}} \approx \frac{\pi}{\sqrt{12}}, \quad \theta_{0}=\frac{\lambda_{0}}{\sqrt{q}} \approx \frac{1}{2^{n}} \frac{\pi}{\sqrt{12}}, \\
w \geq 4, \quad \lambda_{0} \approx \pi \sqrt{2 q} \approx \sqrt{2} \pi 2^{n}, \quad \theta_{0}=\frac{\lambda_{0}}{\sqrt{q}} \approx \sqrt{2} \pi,
\end{array}
$$

where we have inserted $q \approx 4^{n}$ and $\theta_{0}$ is the length of these geodesics in the unpenalized metric $q_{\sigma}=1$.

The geodesics with $w=1$ have a conjugate point after a length which is exponential in $n$. However, this cannot correspond to a cut point. Indeed this conjugate point occurs after that the geodesic has passed through the identity matrix many times, since from Eq. (6.22) we have $\theta_{p} \ll \theta_{0}$ at large $n$.

The geodesics with $w \geq 4$ instead have a conjugate point at $\theta$ of order 1 , with a length which scales exponentially in $n$. If in addition we would know that the cut point coincides with the conjugate point, this would be a proof that maximal complexity is scaling exponentially with $n$. Unfortunately, we do not have a strong indication that this happens. Still, the fact that $\theta$ remains of order 1 makes the possibility that the cut point coincides with conjugate point 
not as unrealistic as in the $w=1$ case. Note that, with very good approximation, there is no dependence on $w$ for $w \geq 4$ in the distance of the conjugate point from the origin.

The geodesics with $w=3$ have a conjugate point at a value of the coordinate $\theta$ very close to the identity. In this limit we have evidence that the conjugate point is also a cut point, because it happens at infinitesimal value of the coordinate $\theta$. However, the distance from the origin is of order 1, so this does not teach us anything interesting about the possible exponential growth of complexity at large $n$. Also, the exponential dependence $\theta \propto 2^{-n}$ shows that draconian penalties are by construction singular.

From the results in Sec. V D, we know that we can find many directions orthogonal to the unbroken subgroup which are also penalties eigenvectors. In particular, all the tangent directions orthogonal to the vertical space with $0 \leq s \leq n-3$, see Eq. (5.31), contain just operators with weight $w \geq 3$ and so generate exponential projectable geodesics. The considerations about conjugate points for these exponential geodesics can then be extended to the state space, with the caveat that the conjugate point might occur before in the state space, see [62].

\section{E. Progressive model}

At leading order in $\alpha$, the Ricci contraction with the unit vector pointing in the direction $\sigma$ is (for $w>1$ )

$$
R_{w}=2 w\left(2^{w-1}-2^{2 n-2 w+1}\right),
$$

and $R_{w}=2$ for $w=1$ [see Appendix B, Eq. (B11)]. This is positive for $w=1$ and for

$$
w>\frac{2}{3}(n+1) .
$$

The conjugate point for $w=1$ is estimated at

$$
\lambda_{0}=\theta_{0}=\frac{\pi}{\sqrt{2}} 2^{n} .
$$

Again, Eq. (6.22) tells us that $\theta_{p} \ll \theta_{0}$ at large $n$, so this conjugate point cannot correspond to a cut point.

The conjugate points for the generators at large $w$ in Eq. (6.26) are more interesting. In this class, the largest positive $R_{w}$ is at $w=n$, which reads

$$
R_{n}=n\left(2^{n}-4\right)
$$

and gives a conjugate point at

$$
\lambda_{0}=\frac{\pi 2^{n / 2}}{\sqrt{n}}, \quad \theta_{0}=\frac{\lambda_{0}}{\alpha^{n / 2}}=\frac{\pi 2^{n / 2}}{\sqrt{n} \alpha^{n / 2}} .
$$

The smallest positive value of $R_{w}$ is realized for slightly different values of the integer $w$, depending on the value of $n$ modulo 3 . We have to distinguish the following cases:

$$
\begin{aligned}
& n=3 a, \quad w=2 \frac{n}{3}+1, \quad R_{w}=2^{2 n / 3}\left(1+\frac{2 n}{3}\right) \approx n 2^{2 n / 3} 0.67, \\
& n=3 a+1, \quad w=\frac{2 n+4}{3}, \quad R_{w}=2^{2 n / 3}(n+2) 2^{1 / 3} \approx n 2^{2 n / 3} 1.26, \\
& n=3 a+2, \quad w=\frac{2 n+5}{3}, \quad R_{w}=2^{2 n / 3} \frac{7(2 n+5)}{6 \cdot 2^{1 / 3}} \approx n 2^{2 n / 3} 1.85,
\end{aligned}
$$

where $a$ is an integer number. In all cases $R_{w} \approx n 2^{2 n / 3}$ up to order one factors. This gives a conjugate point at

$$
\lambda_{0}=\frac{\pi 2^{2 n / 3}}{\sqrt{n}}, \quad \theta_{0}=\frac{\lambda_{0}}{\alpha^{n / 3}}=\frac{\pi 2^{2 n / 3}}{\sqrt{n} \alpha^{n / 3}} .
$$

Intermediate values of the weight give conjugate points distances which scale in between the ones in Eqs. (6.29) and (6.31).

In order to have small $\theta_{0}$ in the large $n$ limit in Eqs. (6.29) and (6.31), we have just to require $\alpha>4$. The required value of $\alpha$ should also be large enough to trust the leading order result (6.25). The Ricci curvature indeed seems to converge to the asymptotic value at large $\alpha$ quite fast (see Fig. 2 for the Ricci scalar).
Since $\theta_{0} \rightarrow 0$, we expect that, for large $n$, the geodesics in Eqs. (6.29) and (6.31) are truly minimizing ones. So we find strong indication that in this limit the distance of the cut point of the geodesics with large $w$ (in the window $\left.\frac{2}{3} n<w<n\right)$ is in between

$$
\frac{\pi 2^{n / 2}}{\sqrt{n}} \leq \lambda_{0} \leq \frac{\pi 2^{2 n / 3}}{\sqrt{n}} .
$$

Consequently, the maximal complexity is bigger than

$$
\lambda_{\max }=\frac{\pi 2^{2 n / 3}}{\sqrt{n}},
$$

and scales exponentially in $n$. 
One may wonder if this is just an artifact of the large $\alpha$ limit: indeed in this regime we expect that the maximal complexity goes to infinity by construction. In order to clarify this subtle point, let us consider higher order corrections to $R_{w}$ and to $\lambda$. The order $\alpha^{-1}$ term vanishes for all the Ricci, except for $w=2$ which is not interesting for conjugate points (see Appendix B). So we need to go to order $\alpha^{-2}$.

To make the computation simpler, let us consider $w=n$. In this case, the nonvanishing $\alpha^{-2}$ terms in the sectional curvatures which contribute to $R_{n}$ are

$$
\begin{aligned}
\delta K(n, 2,0) & =-\frac{3}{\alpha^{2}}, \quad \delta K(n, 3,0)=\frac{2}{\alpha^{2}}, \\
\delta K(n, n-2,0) & =-\frac{2}{\alpha^{2}}, \quad \delta K(n, n-1,0)=\frac{1}{\alpha^{2}}, \\
\delta K(n, N, 1) & =\frac{1}{\alpha^{2}}, \quad \text { for } 4 \leq N \leq n-1 .
\end{aligned}
$$

A direct calculation gives

$$
\begin{aligned}
R_{n} & =n\left(2^{n}-4\right)+\frac{(n-1) n\left(\left(2^{n}-16\right) n-2\left(2^{n}-4\right)\right)}{6 \alpha^{2}}, \\
& \approx n \cdot 2^{n}+\frac{1}{6 \alpha^{2}} n^{3} 2^{n} .
\end{aligned}
$$

The length of the geodesic built from the exponential of a $w=n$ generator before the conjugate point is then, at the next order in $\alpha$ :

$\lambda_{0}=\frac{\pi \cdot 2^{n}}{\sqrt{n \cdot 2^{n}+\frac{n^{3}}{6 \alpha^{2}} 2^{n}}} \approx \frac{\pi}{\sqrt{n}} 2^{n / 2}\left(1-\frac{1}{12 \alpha^{2}} n^{2}\right)$.

In order to trust the approximation, we should just increase $\alpha$ in a way slightly faster than $n$ for large $n$, for example quadratically. From this polynomial increase of $\alpha$ with $n$, we get an exponential increase of complexity. We believe that this is a strong indication that maximal complexity scales exponentially with $n$ with a progressive choice of penalties.

This is not a rigorous proof. For example, we neglected the shear term in the Raychaudhuri equation which may cause the conjugate point to appear before. It would be interesting to improve the analysis studying the impact of these terms. We leave this as a problem for future investigation.

From Sec. V D, we know that for the progressive model there is just a two-dimensional space of vectors, which are both orthogonal to the vertical space and also eigenstates of the penalty. They are generated by arbitrary linear combinations of $S_{n}^{ \pm}$in Eq. (5.28) and they have both $w=n$. So the previous calculation in unitary space for $w=n$ applies also for state complexity, with the caveat that the conjugate point might occur before in the state space, see [62].

\section{CONCLUSIONS}

In this paper we studied several aspects of complexity geometry. Using the formalism introduced in [5] for unitary complexity of a system of $n$ qubits, we showed that the negativity of sectional curvatures $K$ along the directions $\rho$, $\sigma$ in the unitary space is directly related to a large penalty factor for the commutator $[\rho, \sigma]$, i.e.,

$K(\rho, \sigma)=\frac{1}{q_{\rho} q_{\sigma}}\left[-3 q_{[\rho, \sigma]}+2\left(q_{\rho}+q_{\sigma}\right)+\frac{\left(q_{\rho}-q_{\sigma}\right)^{2}}{q_{[\rho, \sigma]}}\right]$.

In this equation, the only negative term is proportional to the penalty of $[\rho, \sigma]$, so that in order to get a negative $K(\rho, \sigma)$ the penalty $q_{[\rho, \sigma]}$ has to dominate compared to $q_{\rho}$ and $q_{\sigma}$; this is always possible for large enough $q_{[\rho, \sigma]}$. From this expression it is clear that negative curvature is always associated to commutators of the form

$$
\text { [easy, easy }]=\text { hard, }
$$

where easy and hard refer to small and large penalty factors respectively. This is consistent with the analysis in [60]. The correlation between negative curvature and the condition in Eq. (7.2) holds also in the qutrit example that we studied in Appendix C.

We applied the formalism of [5] to various examples, both for small and large number of qubits. The one qubit case is already an interesting nutshell for some generic properties (see Sec. III A). First of all, one qubit is a universal closed subsector of the $n$-qubits space, because sectional curvatures inside each qubit space depend just on the penalties of this subsector. In the generic one qubit parameter space, we have that at leas two out of three of the sectional curvatures in the orthogonal basis are positive. This argument shows that, for complexity geometry of $n$ qubits, at least some sectional curvatures are always positive. Moreover, some of the possible behaviors that are realized when some of the penalty factors are sent to infinity generalize to large number of qubits. There are two prototypical situations:

(1) If the easy generators (which are the ones whose penalties are not sent to infinity) are enough to construct the generic unitary, the maximal complexity does not diverge. Some of the sectional curvatures instead diverge and the geometry is singular. An example of this case is realized for $Q \rightarrow \infty$ and $P$ constant.

(2) If the remaining easy generators are not enough to construct the generic unitary, the maximal complexity is infinity by construction and the sectional curvatures do not diverge. An example of this case is for $P=\beta Q \rightarrow \infty$, with $\beta$ constant, where both 
vanishing (for $\beta=1$ ) and negative (for $\beta \neq 1$ ) scalar curvatures can be realized.

For a larger number of qubits $n$ the situation is much more intricate, because the dimension of the space of unitaries scales as $4^{n}$. The allowed values of sectional curvatures in the orthogonal basis have large multiplicities, which can scale exponentially or polynomially with $n$ and the weight $w$. In Appendix A we provide general expressions for this counting. For large $n$ we have a huge arbitrariness in the choice of the penalty factors. Two useful prototypes are

(i) Draconian penalties, defined by Eq. (1.1). In the large $q$ limit, for fixed $n$, complexity does not diverge, and the geometry becomes singular. This is similar to point 1 of the one qubit case.

(ii) Progressive penalties, as defined in Eq. (1.2). In the large $\alpha$ limit, complexity diverges for fixed $n$ and the geometry is not singular (the sectional curvatures scale as $\alpha^{0}$ ). The scalar curvature, see Eq. (4.13), is negative. The situation is similar to point 2 in the one qubit case.

So far we discussed complexity as defined for unitary operators. For applications to holography, it is more relevant to consider the different but somehow related notion of state complexity [8]. Complexity for states is defined as the lowest possible complexity of an operator which prepares the state, starting from a given reference state. In general, we have to minimize over all the possible unitaries that prepare the given state [60]. The complexity metric here is much more intricate, because the geometry is not homogeneous.

In Sec. V, we point out that the relation between the unitary and the state geometry follows directly from the mathematical theory of Riemannian submersions $[61,62,71]$. In particular, the geodesics in the state space $B$ can be found by a projection of a particular class of geodesics (the horizontal ones) from the unitary space $M$. Moreover, conjugate points for geodesics in $B$ are realized for a complexity equal or less than the one in $M$. Curvatures in the state and in the unitary spaces are related by O'Neill's formula [61]. Geodesics in the state space can be in principle computed without even knowing the metric on $B$. Our approach gives also a a closed-form expression for the state metric. We checked that this result reproduces the known 1-qubit metric with arbitrary penalties. As a new application, we determine the state complexity metric and curvatures for the one qutrit example.

An important open problem is to understand the regime in which the complexity metric provides a complexity distance scaling exponentially with the number of qubits. In Sec. VI we provide robust evidence for the exponential behavior of complexity for progressive penalties. The analysis is based on the study of conjugate points in the unitary space. For a general manifold, the study of conjugate points does not provide direct information about the maximal possible complexity, because a geodesic might cross its cut point before the conjugate point. This obstruction can be circumvented if one considers parametric regimes in which the angular position of the conjugate point approaches the identity. In this limit we expect that the cut point coincides with the conjugate point. We show that this regime is realized for progressive penalties at large $\alpha$ and we give an estimate for a lower bound for the scaling of complexity. This bound scales exponentially with $n$.

\section{ACKNOWLEDGMENTS}

We are grateful to Mauro Spera for very precious geometrical insights. We thank Luca Cassia, Alice Gatti and Alessandro Tomasiello for valuable discussions. S. B. acknowledges support from the Independent Research Fund Denmark Grant No. DFF-6108-00340 "Towards a deeper understanding of black holes with non-relativistic holography." A. L. is supported by UKRI Science and Technology Facilities Council (STFC) Consolidated Grants No. ST/P00055X/1 and No. ST/T000813/1. The work of G. B. D. L. is supported in part by the Simons Foundation Origins of the Universe Initiative (modern inflationary cosmology collaboration) and by a Simons Investigator award. N.Z. acknowledges the Ermenegildo Zegna's Group for the financial support.

\section{APPENDIX A: COUNTING NONVANISHING SECTIONAL CURVATURES}

Given two generators $(\rho, \sigma)$, we define $l$ as the number of corresponding tensorial product entries in which $\rho$ and $\sigma$ have different Pauli matrices (for anticommuting $\rho$ and $\sigma, l$ is odd). We define $m$ as the number of corresponding tensorial product entries in which $\rho$ and $\sigma$ have the same Pauli matrices.

The number of entries in the tensorial product in which there is a Pauli matrix in $\sigma$ and an identity in the corresponding entry in $\rho$ is given by

$$
s=N-l-m .
$$

Due to the properties of generalized Pauli matrices, if a pair of generators in the basis do not commute, then they necessarily need to anticommute. Consequently, the commutator $[\rho, \sigma]$ has weight

$$
w=M+N-l-2 m,
$$

where $l+m \leq \min (M, N)$. The minimal weight is realized just for $l=1$ and for $m=\min (N-1, M-1)$. The maximum weight instead is realized by $l=1$ and $m=0$.

In order to parametrize the possible values of the weight $w$, let us introduce an integer label $r$ :

$$
\begin{aligned}
& \text { for } N \leq M \text { : } \\
& r=N-\frac{l+1}{2}-m, \quad r=0, \ldots, N-1,
\end{aligned}
$$




$$
\begin{aligned}
& \text { for } N>M: \\
& r=M-\frac{l+1}{2}-m, \quad r=0, \ldots, M-1,
\end{aligned}
$$

in such a way that the weight of the commutator is

$$
w_{r}=|M-N|+1+2 r .
$$

The $r=0$ case corresponds to the lowest possible weight of the commutator, while the maximum of $r$ corresponds to the maximum weight.

The weight is limited also by the number of qubits, i.e., $w_{r} \leq n$. So, for any given pair $(M, N)$, we must have that the integer $r$ is in the following range:

$$
\begin{aligned}
& \text { for } N \leq M \\
& 0 \leq r \leq \min \left(N-1, \frac{n-|M-N|-1}{2}\right),
\end{aligned}
$$

for $N>M$,

$$
0 \leq r \leq \min \left(M-1, \frac{n-|M-N|-1}{2}\right) \text {. }
$$

Note that for each fixed number of qubits $n, r \leq$ $[(n-1) / 2]$ where $[\ldots]$ denotes the integer part.

Given a generator $\rho$ in the basis with weight $M$, we similarly denote by $\mathcal{R}(M, N, r)$ the number of generators with weight $N$ whose commutator with $\rho$ has a weight parametrized by a given integer $r$, as in Eq. (A5).

We give now an explicit formula for $\mathcal{R}(M, N, r)$. Let us first consider the $N \leq M$ case and let us start with $r=0$. In this case we need to determine how many $\sigma$ will give a $[\rho, \sigma]$ with the minimal possible weight. As stressed before, this is realized just for $l=1, m=N-1$, and $s=0$. We have $M$ places to stick the $l=1$ entry of $\sigma$ (which corresponds to a different Pauli matrix compared to $\rho$, so there is an extra factor of 2), and then we have $\left(\begin{array}{c}M-1 \\ N-1\end{array}\right)$ ways to stick the $m=N-1$ entries of $\sigma$ with the same Pauli matrix as in $\rho$. The number of such matrices is

$$
\mathcal{R}(M, N, 0)=2 M\left(\begin{array}{c}
M-1 \\
N-1
\end{array}\right) .
$$

Let us consider $r=1$. Here in general we have two possible situations. We may have $l=1, m=N-2, s=1$ or instead $l=3, m=N-3$, and $s=0$. In the first case, there are three ways to choose the Pauli matrix in $\sigma$ which has an identity in the corresponding entry in $\rho$. This gives

$$
\begin{aligned}
\mathcal{R}(M, N, 1)= & \left(\begin{array}{c}
M \\
3
\end{array}\right) 2^{3}\left(\begin{array}{c}
M-3 \\
N-3
\end{array}\right) \\
& +\left(\begin{array}{c}
M \\
1
\end{array}\right) 2^{1}\left(\begin{array}{c}
M-1 \\
N-2
\end{array}\right)\left(\begin{array}{c}
n-M \\
1
\end{array}\right) 3 .
\end{aligned}
$$

In the general case we have to sum over all the possible odd values of $l$; it is then convenient to set $l=2 k+1$ with integer $k$. In general we have $\left(\begin{array}{c}M \\ l\end{array}\right) 2^{l}$ ways to set the entries in tensor product where $\rho$ and $\sigma$ have different Pauli matrices, $\left(\begin{array}{c}M-l \\ m\end{array}\right)$ ways to set the entries in such a way that $\rho$ and $\sigma$ have the same Pauli matrices in the corresponding entries and $\left(\begin{array}{c}n-M \\ s\end{array}\right) 3^{s}$ ways to set entries in which in the corresponding elements of $\rho$ and $\sigma$ there are an identity matrix and a Pauli matrix, respectively. The total combinatorial factors is

$$
\begin{aligned}
\mathcal{R}(M, N, r)= & \sum_{k=0}^{r}\left(\begin{array}{c}
M \\
l
\end{array}\right) 2^{l}\left(\begin{array}{c}
M-l \\
m
\end{array}\right)\left(\begin{array}{c}
n-M \\
s
\end{array}\right) 3^{s} \\
= & \sum_{k=0}^{r}\left(\begin{array}{c}
M \\
2 k+1
\end{array}\right) 2^{2 k+1}\left(\begin{array}{c}
M-2 k-1 \\
N-k-1-r
\end{array}\right) \\
& \times\left(\begin{array}{c}
n-M \\
r-k
\end{array}\right) 3^{r-k},
\end{aligned}
$$

where we used $s=r-k$. In this expression we should not worry about negative values of $N-k-1-r$, which indeed may occur, because the corresponding terms in the sum vanish after analytically continuing the binomial coefficients with the $\Gamma$ function.

If $N>M$, we can write a similar formula. We can still use the same Eq. (A10), with $s=r-k+N-M$ and $m$ accordingly given by (A1):

$$
\begin{aligned}
\mathcal{R}(M, N, r)= & \sum_{k=0}^{r}\left(\begin{array}{c}
M \\
l
\end{array}\right) 2^{l}\left(\begin{array}{c}
M-l \\
m
\end{array}\right)\left(\begin{array}{c}
n-M \\
s
\end{array}\right) 3^{s} \\
= & \sum_{k=0}^{r}\left(\begin{array}{c}
M \\
2 k+1
\end{array}\right) 2^{2 k+1}\left(\begin{array}{c}
M-2 k-1 \\
M-k-1-r
\end{array}\right) \\
& \times\left(\begin{array}{c}
n-M \\
r-k+N-M
\end{array}\right) 3^{r-k+N-M} .
\end{aligned}
$$

Let us denote by $\mathcal{N}(M, N, r)$ the number of sectional curvatures with value given by Eq. (4.4). These can be found as

$\mathcal{N}(M, N, r)=\mathcal{N}_{M} \mathcal{R}(M, N, r)=\mathcal{N}_{N} \mathcal{R}(N, M, r)$,

where $\mathcal{N}_{M}, \mathcal{N}_{N}$ are defined in Eq. (4.1).

\section{APPENDIX B: EXPLICIT CALCULATIONS FOR THE PROGRESSIVE PENALTIES CASE}

In this section we consider the choice in Eq. (4.11). A direct calculation gives, for $N \leq M$ :

$$
\begin{aligned}
K(M, N, r)= & -3 \alpha^{2(r+1-N)}+2 \alpha^{1-N}+2 \alpha^{1-M} \\
& +\alpha^{-2 r}\left(1+\alpha^{-2(M-N)}-2 \alpha^{-(M-N)}\right)
\end{aligned}
$$

and, for $N>M$ : 


$$
\begin{aligned}
K(M, N, r)= & -3 \alpha^{2(r+1-M)}+2 \alpha^{1-N}+2 \alpha^{1-M} \\
& +\alpha^{-2 r}\left(1+\alpha^{-2(N-M)}-2 \alpha^{-(N-M)}\right) .
\end{aligned}
$$

Note that at large $\alpha$ sectional curvatures scale at most as $\alpha^{0}+\mathcal{O}\left(\alpha^{-1}\right)$.

\section{Leading order}

Let us start with the $\alpha^{0}$ terms. For $r=0$, the only nonvanishing sectional curvatures at this order are $K=1$, for $M=N=1$ and

$$
M, N>1, \quad M \neq N \text {. }
$$

For $r \geq 1$, the only term that can be of order $\alpha^{0}$ is for $M, N>1$ and is given by

$K(M, N, r)=\left\{\begin{array}{ll}-3 \alpha^{2(r+1-N)}+O\left(\alpha^{-1}\right) & M \geq N \\ -3 \alpha^{2(r+1-M)}+O\left(\alpha^{-1}\right) & M<N\end{array}\right.$.

If $M \geq N$, we have $r=0, \ldots, N-1$, then $K=-3$ only for the maximal value $r=N-1$. If $M<N$, we have $r=0, \ldots, M-1$, then $K=-3$ only for the maximal value $r=M-1$.

We first compute the Ricci tensor contracted with a unit vector $u(\sigma)$, where $\sigma$ has weight $M$, as defined in Eq. (2.20):

$$
R_{M}=\sum_{N} \sum_{r} K(M, N, r) \mathcal{R}(M, N, r)
$$

For $M=1$, the only leading-order contribution is for $M=N=1$ :

$$
R_{1}=\mathcal{R}(1,1,0)=2 .
$$

Let us now consider $1<M \leq n$. The positive leading-order contributions to $R_{M}$ are given by the scalar curvatures with $r=0$, whose value is $K=1$ :

$$
\begin{aligned}
R_{M}^{+} & =\sum_{N=2}^{M-1} \mathcal{R}(M, N, 0)+\sum_{N=M+1}^{n} \mathcal{R}(M, N, 0) \\
& =2 M\left(2^{M-1}-3+2^{2(n-M)}\right)
\end{aligned}
$$

The negative leading-order contributions to $R_{M}$ are given by the scalar curvatures with $r=N-1$ if $M \geq N$ and $r=$ $M-1$ if $M<N$, all equal to $K=-3$. The expression turns out to be the same for both the cases:

$$
\begin{aligned}
& \text { for } M \geq N \\
& \mathcal{R}(M, N, N-1)=2 M\left(\begin{array}{c}
n-M \\
N-1
\end{array}\right) 3^{N-1},
\end{aligned}
$$

$$
\begin{aligned}
& \text { for } M<N \\
& \mathcal{R}(M, N, M-1)=2 M\left(\begin{array}{c}
n-M \\
N-1
\end{array}\right) 3^{N-1},
\end{aligned}
$$

We finally get

$$
\begin{aligned}
R_{M}^{-} & =\sum_{N=2}^{1+n-M} 2 M\left(\begin{array}{c}
n-M \\
N-1
\end{array}\right) 3^{N-1}, \\
& =-6 M\left[2^{2(n-M)}-1\right] .
\end{aligned}
$$

The maximum value of $N$ in the sum, $N_{\max }=1+n-M$, ensures that $r=M-1$ is allowed in the case $M<N$, as can be obtained from Eq. (A6).

The final result for $R_{M}$ at the leading order is

$$
R_{M}=R_{M}^{+}+R_{M}^{-}=2 M\left(2^{M-1}-2^{2(n-M)+1}\right)
$$

Using Eq. (4.1) and this result, the scalar curvature is computed as

$$
R=\sum_{M=1}^{n} \mathcal{N}_{M} R_{M}=3 n\left(4^{n}-27^{n-1}\right)
$$

\section{Next-to-leading order}

We can systematically improve this calculation order by order in the expansion parameter $\alpha$. For example, at order $\alpha^{-1}$, the only nonzero contribution to the sectional curvatures, which we denote as $\delta K(M, N, r)$, are

$$
\begin{aligned}
& M=N=2, \quad r=0,1, \quad \delta K=\frac{4}{\alpha}, \\
& M=N+1, \quad N \geq 3, \quad r=0, \quad \delta K=-\frac{2}{\alpha}, \\
& M=2, \quad N \geq 4, \quad r=0, \quad \delta K=\frac{2}{\alpha}, \\
& M=2, \quad N \geq 3, \quad r=1, \quad \delta K=\frac{2}{\alpha},
\end{aligned}
$$

and the ones obtained exchanging $M$ with $N$. Due to a nontrivial cancellation, the only corrections to $R_{M}$ is for $w=2$

$$
\delta R_{2}=\frac{4^{n}}{\alpha}
$$

This gives the following correction to the curvature

$$
\delta R=\frac{9}{2} n(n-1) \frac{4^{n}}{\alpha} .
$$




\section{APPENDIX C: STATE COMPLEXITY FOR ONE QUTRIT}

In this section we show an application of the method in Sec. V B to determine the metric and the curvature properties in the space of states, using the explicit decomposition of the unitary space as a submersion. We consider the case of a qudit theory, which describes a system with $n$ energy levels. In particular, we focus on the case of one qutrit, where $n=3$ and the group manifold is $M=S U(3)$.

The corresponding space of states is $M / G=\mathbb{C P}^{2}$, which is parametrized by two complex coordinates $\left(z_{i}, \bar{z}_{i}\right)$ with $i \in\{1,2\}$. Alternatively, we can use four real coordinates $\left(\theta_{i}, \phi_{i}\right)$ where $\theta_{i} \in[0, \pi]$ and $\phi_{i} \in[0,2 \pi]$ with $i \in\{1,2\}$. The parametrization with complex coordinates is useful to transform the reference state, which we conventionally take to be $\left|\psi_{0}\right\rangle=(1,0,0)$, into the generic state

$$
|\psi\rangle=\frac{1}{\sqrt{1+z_{i} \bar{z}^{i}}}\left(\begin{array}{c}
1 \\
z_{1} \\
z_{2}
\end{array}\right)=\left(\begin{array}{c}
\cos \theta_{1} \\
e^{i \phi_{1}} \sin \theta_{1} \cos \theta_{2} \\
e^{i \phi_{2}} \sin \theta_{1} \sin \theta_{2}
\end{array}\right) .
$$

The parametrization with angular coordinates, which we use in the second equality, will be convenient to describe the curvatures, giving a compact expression for the Ricci scalar.

Here and in the following, the subscript refers to the coordinate dependence of the group element from the space of states $(\theta$ subscript $)$ or from the additional coordinates that bring to the space of unitaries ( $\lambda$ subscript). Instead the superscript $(K)$ refers to the group $S U(K)$ to which the element belongs. The generic element of the coset space $M / G$ is given by

$$
\begin{aligned}
U_{\theta}^{(3)} & =\frac{1}{\sqrt{1+z_{i} \bar{z}^{i}}}\left(\begin{array}{ccc}
1 & -\bar{z}_{j} \\
z_{i} & \sqrt{1+z_{i} \bar{z}^{i}} \delta_{i j}-\frac{z_{i} \bar{z}_{j}}{1+\sqrt{1+z_{i} \bar{z}^{i}}}
\end{array}\right) \\
& =\left(\begin{array}{ccc}
\cos \theta_{1} & -e^{-i \phi_{1}} \sin \theta_{1} \cos \theta_{2} & -e^{-i \phi_{2}} \sin \theta_{1} \sin \theta_{2} \\
-e^{i \phi_{1}} \sin \theta_{1} \cos \theta_{2} & \cos \left(\frac{\theta_{1}^{2}}{2}\right)-\cos \left(2 \theta_{2}\right) \sin \left(\frac{\theta_{1}^{2}}{2}\right) & -e^{i\left(\phi_{1}-\phi_{2}\right)} \sin \left(\frac{\theta_{1}^{2}}{2}\right) \sin \left(2 \theta_{2}\right) \\
-e^{i \phi_{2}} \sin \theta_{1} \sin \theta_{2} & -e^{-i\left(\phi_{1}-\phi_{2}\right)} \sin \left(\frac{\theta_{1}^{2}}{2}\right) \sin \left(2 \theta_{2}\right) & \cos \left(\frac{\theta_{1}^{2}}{2}\right)+\cos \left(2 \theta_{2}\right) \sin \left(\frac{\theta_{1}^{2}}{2}\right)
\end{array}\right) .
\end{aligned}
$$

While the last equality is specific of this case, the expression in the first line applies to the space $\mathbb{C P}^{K}$ with $K \in \mathbb{N}$ arbitrary. In the general case, the only difference is that the index runs over $i \in\{1, \ldots, K\}$.

The group $S U(3)$ contains as maximal subgroup $S U(2) \times U(1)$. In order to build the stabilizer of the element $(1,0,0)$ inside $S U(3)$, we use a recursive procedure. The $S U(2)$ factor corresponds to the case of a single qubit: then the stabilizer of the element $(1,0)$ is given by the exponential of the Pauli matrix $\sigma_{z}$, which reads

$$
V_{\lambda}^{(2)}=e^{i \lambda_{2} \sigma_{z}}=\left(\begin{array}{cc}
e^{i \lambda_{2}} & 0 \\
0 & e^{-i \lambda_{2}}
\end{array}\right)
$$

Now we consider the coset element of $S U(2)$, that can be easily taken from the lower-dimensional generalization of Eq. (C2) and reads

$$
U_{\lambda}^{(2)}=\left(\begin{array}{cc}
\cos \lambda_{1} & -e^{-i \lambda_{3}} \sin \lambda_{1} \\
e^{i \lambda_{3}} \sin \lambda_{1} & \cos \lambda_{1}
\end{array}\right) .
$$

In this way we build the generic element of $S U(2)$ as

$$
\begin{aligned}
U^{(2)} & =U_{\lambda}^{(2)} V_{\lambda}^{(2)}, \\
& =\left(\begin{array}{cc}
e^{i \lambda_{2}} \cos \lambda_{1} & -e^{-i\left(\lambda_{2}+\lambda_{3}\right)} \sin \lambda_{1} \\
e^{i\left(\lambda_{2}+\lambda_{3}\right)} \sin \lambda_{1} & e^{-i \lambda_{2}} \cos \lambda_{1}
\end{array}\right) .
\end{aligned}
$$

Finally, the stabilizer of the reference state inside $S U(3)$ requires another $U(1)$ factor, coming from a global phase that does not change the physics of the system. Indeed, we have the freedom to add another real variable, and the generic element of the maximal subgroup can be written as

$$
V_{\lambda}^{(3)}=p_{2} U_{E}^{(2)},
$$

with the phasis given by the matrix

$$
p_{K}=\left(\begin{array}{cccc}
e^{i K \lambda_{2 K}} & 0 & \cdots & 0 \\
0 & e^{-i \lambda_{2 K}} & \cdots & \cdots \\
\cdots & \cdots & \cdots & 0 \\
0 & \cdots & 0 & e^{-i \lambda_{2 K}}
\end{array}\right) \text {, }
$$

and where we need to embed the matrix $U^{(2)}$ inside $S U(3)$ as follows:

$$
U_{E}^{(2)} \equiv\left(\begin{array}{cc}
1 & 0 \\
0 & U^{(2)}
\end{array}\right)
$$

In this way we finally obtain the stabilizer of the reference state as 


$$
V_{\lambda}^{(3)}=\left(\begin{array}{ccc}
e^{2 i \lambda_{4}} & 0 & 0 \\
0 & e^{i\left(\lambda_{2}-\lambda_{4}\right)} \cos \lambda_{1} & -e^{-i\left(\lambda_{2}+\lambda_{3}+\lambda_{4}\right)} \sin \lambda_{1} \\
0 & e^{i\left(\lambda_{2}+\lambda_{3}-\lambda_{4}\right)} \sin \lambda_{1} & e^{-i\left(\lambda_{2}+\lambda_{4}\right)} \cos \lambda_{1}
\end{array}\right) .
$$

It depends on four real coordinates $\lambda_{i}$, with $i \in\{1,2,3,4\}$.

Now we want to apply Eq. (5.21) to determine the metric on the states space starting from the right-invariant form $u_{s}$ and the left-invariant form $v_{s}$ defined in (5.18). In addition, we need to specify the penalty matrix $M$. The most relevant case corresponds to penalizing the unbroken generators, because it is a configuration that allows for the existence of commutators of the form

$$
\text { [easy, easy }]=\text { hard, }
$$

which are expected to generate negative curvature. This happens due to the algebraic relations (3.14), which occur because we selected a maximal subalgebra. In addition, by considering $0 \leq P<1$, we can also realize a relation of the form (3.12), where only the broken generators are penalized.

For these reasons, we take the penalty matrix to be

$$
M=\operatorname{diag}(P, P, P, P, 1,1,1,1),
$$

where the first four components refer to directions along the maximal subgroup $\mathrm{SU}(2) \times \mathrm{U}(1)$, and the last four directions to the broken generators.

We analytically compute the metric on states (5.21). The result is

$$
\begin{aligned}
d s_{S}^{2}= & d \theta_{1}^{2}+\frac{2 P \sin ^{2} \theta_{1}}{A\left(\theta_{1}\right)} d \theta_{2}^{2}+\frac{2 P \sin ^{2} \theta_{1} \cos ^{2} \theta_{2}}{A\left(\theta_{1}\right)} d \phi_{1}^{2} \\
& +\frac{C\left(\theta_{1}, \theta_{2}\right)}{A\left(\theta_{1}\right) B\left(\theta_{1}\right)} d \phi_{2}^{2}+\frac{2 P \sin ^{2} \theta_{1} \cos ^{2} \theta_{2}}{A\left(\theta_{1}\right) B\left(\theta_{1}\right)} D\left(\theta_{1}\right) \\
& \times\left(\cos ^{2} \theta_{2}\left(d \phi_{1}-d \phi_{2}\right)^{2}+2 d \phi_{1} d \phi_{2}\right),
\end{aligned}
$$

where we defined for convenience the quantities

$$
\begin{aligned}
A\left(\theta_{1}\right) \equiv & (P-1) \cos \left(2 \theta_{1}\right)+P+1, \\
B\left(\theta_{1}\right) \equiv & (P-1) \cos \left(4 \theta_{1}\right)+P+1, \\
C\left(\theta_{1}, \theta_{2}\right) \equiv & P \sin ^{2} \theta_{1}\left[B\left(\theta_{1}\right)-\cos \left(2 \theta_{2}\right)\right. \\
& \left.\times\left(2 P \cos \left(2 \theta_{1}\right)+(P-1) \sin ^{2}\left(2 \theta_{1}\right)\right)\right], \\
D\left(\theta_{1}\right) \equiv & 3(P-1) \cos \left(2 \theta_{1}\right)+P+3 .
\end{aligned}
$$

The metric depends on the angles $\theta_{i}$ but not on the phases $\phi_{i}$. The scalar curvature reads

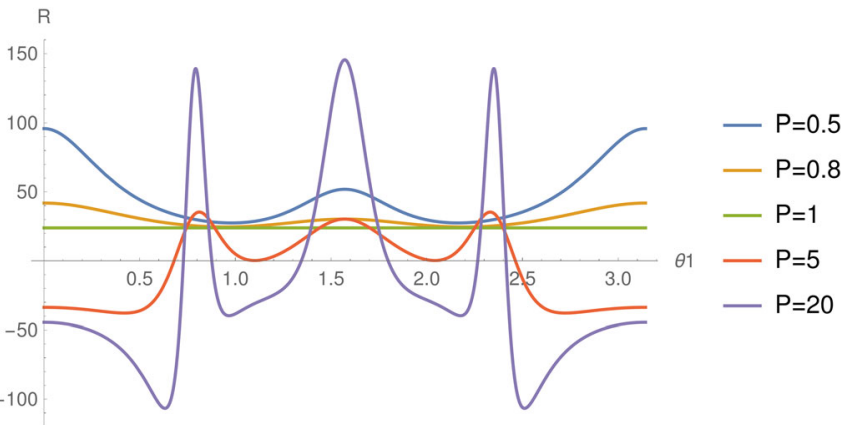

FIG. 6. Scalar curvature (C13) for the state space $\mathbb{C P}^{2}$ with penalty factors $P$ applied to all the generators of the maximal subgroup.

$$
\begin{aligned}
R= & \frac{15}{2}\left(\frac{1}{P}-1\right)+\frac{14 P}{\left((P-1) \cos ^{2} \theta_{1}+1\right)^{2}} \\
& +\frac{2-2 P(3 P+14)}{(P+3)(P-1) \cos ^{2} \theta_{1}+P+3} \\
& +\frac{96 P}{\left((P-1) \cos \left(4 \theta_{1}\right)+P+1\right)^{2}} \\
& +\frac{-8(P-1)(9 P+19) \cos ^{2} \theta_{1}+3 P(P-18)+3}{(P+3)\left((P-1) \cos \left(4 \theta_{1}\right)+P+1\right)} .
\end{aligned}
$$

We observe that the Ricci scalar depends only on the angular coordinate $\theta_{1}$, giving a further simplification with respect to the metric on $\mathbb{C P}^{2}$. This is due to the many symmetries of the penalties in Eq. (C10).

In Fig. 6 we plot the Ricci scalar as a function of $\theta_{1}$ for different values of the penalty $P$. We observe that when $0<P<1$ the scalar curvature is always positive, and reaches a constant value $R=24$ when $P=1$, the case of undeformed inner product on $S U(3)$. When $P>1$ there is always a region with negative curvature, which increases its size accordingly to the increasing of the penalty.

We consider the limit when $P \rightarrow \infty$, which means that the motion along the subgroup directions is strongly penalized. In this limit the Ricci scalar is

$$
\begin{aligned}
\lim _{P \rightarrow \infty} R= & -\frac{3}{2}\left[\sec \left(2 \theta_{1}\right)\left(11 \sec \left(2 \theta_{1}\right)+12\right)\right. \\
& \left.+4 \sec ^{2} \theta_{1}+5\right] .
\end{aligned}
$$

As can be seen in Fig. 7, in such a case the Ricci scalar is always negative and contains singularities. In the opposite limit $P \rightarrow 0$ we instead obtain everywhere a positive and divergent Ricci scalar, since it contains a singular term proportional to $P^{-1}$.

The behavior of the curvature in this example is similar to the one qubit case with $Q=1$ and $P$ generic, which was studied in detail in [60]. 


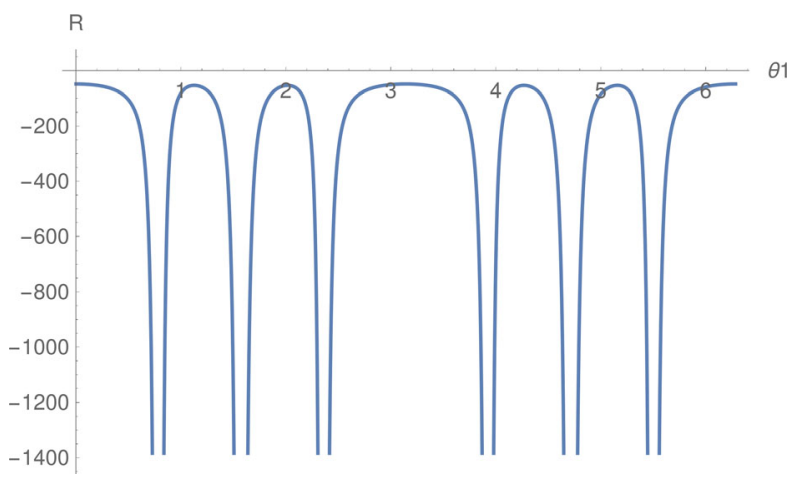

FIG. 7. Scalar curvature (C14) for the unitary space $S U(3)$ in the limiting case of penalty $P \rightarrow \infty$ applied to all the generators of the maximal subgroup.

\section{APPENDIX D: SUBMERSION FOR ONE QUBIT}

Let us apply the method of submersion to the one qubit case. In order to generate a state specified by the $(\theta, \phi)$ angles on the Bloch sphere starting from $|0\rangle$, we can use the following unitary

$$
U_{\theta}=\exp \left[\frac{i \theta}{2}\left(\sigma_{x} \cos \phi+\sigma_{y} \sin \phi\right)\right] .
$$

The action of unbroken generators can be parametrized by $V_{\lambda}$

$$
V_{\lambda}=\exp \left(i \frac{\sigma_{z}}{2} \lambda\right)
$$

and the generic $S U(2)$ transformation is

$$
U=U_{\theta} V_{\lambda}=\left(\begin{array}{cc}
e^{\frac{i \lambda}{2}} \cos \left(\frac{\theta}{2}\right) & i \sin \left(\frac{\theta}{2}\right) e^{-\frac{1}{2} i(\lambda+2 \phi)} \\
i \sin \left(\frac{\theta}{2}\right) e^{\frac{1}{2}(\lambda+2 \phi)} & e^{-\frac{i \lambda}{2}} \cos \left(\frac{\theta}{2}\right)
\end{array}\right) .
$$

The submersion is realized by the projection

$$
\pi: \quad(\lambda, \theta, \phi) \rightarrow(\theta, \phi),
$$

and the vertical space is spanned by $\partial_{\lambda}$.

The metric on the unitary space $M$, with penalties $P$ and $Q$ as in Sec. III A, is

$$
\begin{aligned}
d s^{2}= & \frac{1}{4}\left\{\left(\operatorname{Tr}\left[i d U U^{\dagger} \sigma_{x}\right]\right)^{2}\right. \\
& \left.+Q\left(\operatorname{Tr}\left[i d U U^{\dagger} \sigma_{y}\right]\right)^{2}+P\left(\operatorname{Tr}\left[i d U U^{\dagger} \sigma_{z}\right]\right)^{2}\right\},
\end{aligned}
$$

where

$$
d U=\frac{\partial U}{\partial \theta} d \theta+\frac{\partial U}{\partial \phi} d \phi+\frac{\partial U}{\partial \lambda} d \lambda .
$$

Explicitly, we find

$$
d U U^{\dagger}=i\left(\begin{array}{cc}
a & b \\
b^{*} & -a
\end{array}\right) .
$$

where

$$
\begin{aligned}
& a=\frac{1}{2}((d \lambda+d \phi) \cos \theta-d \phi), \\
& b=\frac{1}{2} e^{-i \phi}(d \theta-i(d \lambda+d \phi) \sin \theta) .
\end{aligned}
$$

Using the unitary metric, we can find the horizontal vectors fields (which are defined as orthogonal to the vertical direction $\partial_{\lambda}$ )

$$
\begin{aligned}
& h_{1}=\partial_{\theta}-\frac{(Q-1) \sin \theta \sin 2 \phi}{2\left(P \cos ^{2} \theta+\sin ^{2} \theta\left(Q \cos ^{2} \phi+\sin ^{2} \phi\right)\right)} \partial_{\lambda}, \\
& h_{2}=\partial_{\phi}+\frac{-2 P \cos ^{2} \theta+2 P \cos \theta-\sin ^{2} \theta((Q-1) \cos 2 \phi+Q+1)}{2\left(P \cos ^{2} \theta+\sin ^{2} \theta\left(Q \cos ^{2} \phi+\sin ^{2} \phi\right)\right)} \partial_{\lambda},
\end{aligned}
$$

which have the property $\pi\left(h_{1}\right)=\partial_{\theta}, \pi\left(h_{2}\right)=\partial_{\phi}$.

Then we can use Eq. (5.22) to find the curvature in the states space, using the results for the one qubit unitaries in Sec. III A. An explicit calculation gives the curvature in the states space:

$$
R=\frac{\alpha}{\beta},
$$

where

$$
\begin{aligned}
\alpha= & 8\left\{-2(Q-1) \sin ^{2} \theta \cos ^{2} \phi\right. \\
& \times\left[-P^{2}+(P-1) \cos ^{2} \theta(P-Q)^{2}+P+Q^{2}\right] \\
& +(P-1) \cos ^{2} \theta\left[-2\left(P^{2}-Q^{2}+Q\right)\right. \\
& \left.-(P-1)(Q-1)(P-Q) \cos ^{2} \theta\right] \\
& +(P-1)(Q-1)^{2}(P-Q) \sin ^{4} \theta \cos ^{4} \phi \\
& \left.+P(Q-1)+(Q-1) Q+P^{2}\right\},
\end{aligned}
$$




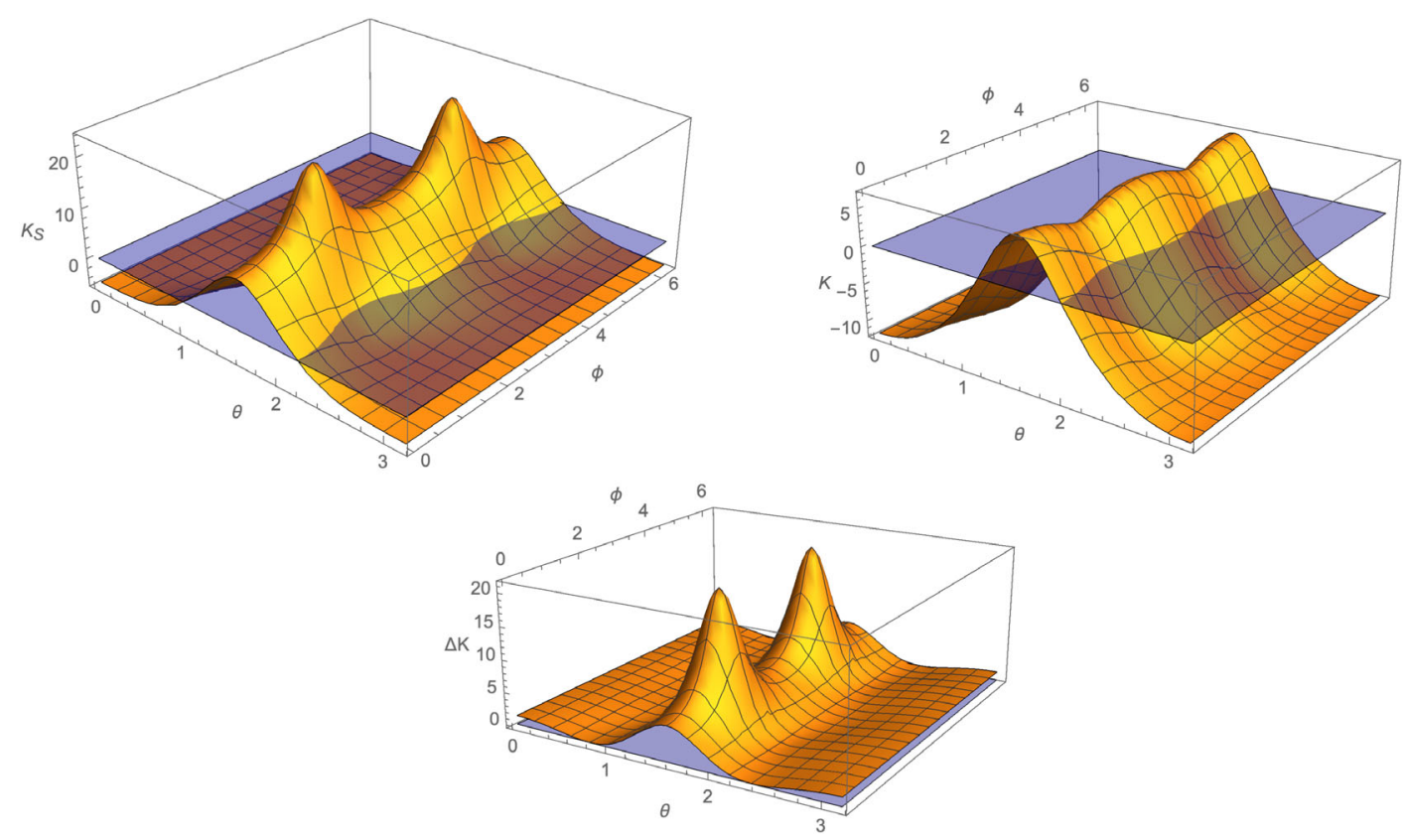

FIG. 8. Comparison of $K_{S}\left(\tilde{h}_{1}, \tilde{h}_{2}\right), K\left(h_{1}, h_{2}\right)$, and $\Delta K$ as a function of $(\theta, \phi)$. The numerical values $P=6, Q=3$ have been used for illustrative purposes.

$$
\begin{aligned}
\beta= & P Q\left[(P-1) \cos ^{2} \theta\right. \\
& \left.+(Q-1) \sin ^{2} \theta \cos ^{2} \phi+1\right]^{2},
\end{aligned}
$$

which matches with the one that can be calculated directly from the states metric in [60]. The difference of the sectional curvatures between the unitary and the state spaces matches with O'Neill formula

$$
\begin{aligned}
\Delta K & =K_{S}\left(\tilde{h}_{1}, \tilde{h}_{2}\right)-K\left(h_{1}, h_{2}\right), \\
& =\frac{3}{4} \frac{\left|\mathcal{V}\left(\left[h_{1}, h_{2}\right]\right)\right|^{2}}{\left|h_{1}\right|^{2}\left|h_{2}\right|^{2}-\left\langle h_{1}, h_{2}\right\rangle^{2}} .
\end{aligned}
$$

The plot of $K_{S}\left(\tilde{h}_{1}, \tilde{h}_{2}\right), K\left(h_{1}, h_{2}\right)$ and $\Delta K$ for particular values of the penalties is shown in Fig. 8.

\section{APPENDIX E: SHEAR TENSOR EQUATION}

The equation for the traceless part $\sigma_{\alpha \beta}$ in Euclidean signature is

$$
\begin{aligned}
\frac{D \sigma_{\mu \nu}}{d \lambda}= & -\frac{2}{d-1} \Theta \sigma_{\mu \nu}-\sigma_{\mu}^{\sigma} \sigma_{\nu \sigma} \\
& +\frac{1}{d-1} h_{\mu \nu} \sigma^{\alpha \beta} \sigma_{\alpha \beta}-C_{\mu \alpha \nu \beta} u^{\alpha} u^{\beta}-\frac{1}{d-2} \bar{R}_{\mu \nu},
\end{aligned}
$$

where

$$
\bar{R}_{\mu \nu}=h_{\mu}^{\alpha} h_{\nu}^{\beta} R_{\alpha \beta}-\frac{1}{(d-1)} R_{\alpha \beta} h^{\alpha \beta} h_{\mu \nu}
$$

is the projected trace-free part of $R_{\mu \nu}$.
The Weyl tensor is given by

$$
\begin{aligned}
C_{\mu \alpha \nu \beta}= & -R_{\mu \alpha \nu \beta}+\frac{R_{\mu \beta} g_{\alpha \nu}-R_{\mu \nu} g_{\alpha \beta}+R_{\alpha \nu} g_{\mu \beta}-R_{\alpha \beta} g_{\mu \nu}}{d-2} \\
& +\frac{g_{\mu \nu} g_{\alpha \beta}-g_{\mu \beta} g_{\alpha \nu}}{(d-1)(d-2)} R,
\end{aligned}
$$

and its contraction with the normalized velocity is

$$
C_{\mu \alpha \nu \beta} u^{\alpha}(\sigma) u^{\beta}(\sigma)=\frac{1}{q_{\sigma}} C_{\mu \sigma \nu \sigma} .
$$

Recalling that in our basis $R_{\mu \sigma \nu \sigma}=-R_{\mu \sigma \sigma \nu} \neq 0$ only if $\mu=$ $\nu$ and that both the metric and the Ricci tensor are diagonal, we conclude that $C_{\mu \sigma \nu \sigma} \neq 0$ only if $\mu=\nu$. However, if $\mu=\nu=\sigma$, we have $C_{\mu \sigma \nu \sigma}=0$. Therefore, the only relevant nonvanishing components of $C_{\mu \sigma \nu \sigma}$ are the ones with $\mu=\nu=\rho \neq \sigma$. These components read

$$
C_{\rho \sigma \rho \sigma}=q_{\rho} q_{\sigma}\left[K(\rho, \sigma)-\frac{R_{\rho}+R_{\sigma}}{d-2}+\frac{R}{(d-1)(d-2)}\right] .
$$

The only nonvanishing components of the Weyl tensor contraction with the normalized velocity are the ones with $\rho \neq \sigma$ :

$$
\begin{aligned}
& C_{\rho \alpha \rho \beta} u^{\alpha}(\sigma) u^{\beta}(\sigma) \\
& \quad=q_{\rho}\left[K(\rho, \sigma)-\frac{R_{\rho}+R_{\sigma}}{d-2}+\frac{R}{(d-1)(d-2)}\right]
\end{aligned}
$$


A direct calculation gives that $\bar{R}_{\mu \nu}$ is nonvanishing only if $\mu=\nu=\rho \neq \sigma$ :

$$
\bar{R}_{\rho \rho}=q_{\rho}\left[R_{\rho}-\frac{1}{d-1}\left(R-R_{\sigma}\right)\right] .
$$

The nonvanishing components of the tensor entering into the shear equation (E1) are thus the ones with $\rho \neq \sigma$ :

$$
C_{\rho \alpha \rho \beta} u^{\alpha}(\sigma) u^{\beta}(\sigma)+\frac{1}{d-2} \bar{R}_{\rho \rho}=q_{\rho}\left\{K(\rho, \sigma)-\frac{1}{d-1} R_{\sigma}\right\} .
$$

Note that in the one qubit case $(d=3)$, by means of Eqs. (3.1) and (6.15), all the components of the above tensor vanish for $G_{x}$ if $P=Q$, for $G_{y}$ if $P=1$ and for $G_{z}$ if $Q=1$. In these cases, from Eq. (E1) we get that if the shear tensor $\sigma_{\alpha \beta}=0$, then it vanishes along all the geodesic.
[1] M. A. Nielsen, A geometric approach to quantum circuit lower bounds, Quantum Inf. Comput. 6, 213 (2006).

[2] M. A. Nielsen, M. Dowling, M. Gu, and A. C. Doherty, Quantum computation as geometry, Science 311, 1133 (2006).

[3] M. A. Nielsen, M. Dowling, M. Gu, and A. C. Doherty, Optimal control, geometry, and quantum computing, Phys. Rev. A 73, 062323 (2006).

[4] M. Gu, A. C. Doherty, and M. A. Nielsen, Quantum control via geometry: An explicit example, Phys. Rev. A 78, 032327 (2008).

[5] M. R. Dowling and M. A. Nielsen, The geometry of quantum computation, Quantum Inf. Comput. 8, 0861 (2008).

[6] L. Susskind, Computational complexity and black hole horizons, Fortschr. Phys. 64, 24 (2016); 64, 44(A) (2016).

[7] D. Stanford and L. Susskind, Complexity and shock wave geometries, Phys. Rev. D 90, 126007 (2014).

[8] L. Susskind, Entanglement is not enough, Fortschr. Phys. 64, 49 (2016).

[9] L. Susskind, The typical-state paradox: Diagnosing horizons with complexity, Fortschr. Phys. 64, 84 (2016).

[10] L. Susskind, Three lectures on complexity and black holes, arXiv:1810.11563.

[11] S. Ryu and T. Takayanagi, Holographic Derivation of Entanglement Entropy from AdS/CFT, Phys. Rev. Lett. 96, 181602 (2006).

[12] D. N. Page, Information in Black Hole Radiation, Phys. Rev. Lett. 71, 3743 (1993).

[13] A. Almheiri, N. Engelhardt, D. Marolf, and H. Maxfield, The entropy of bulk quantum fields and the entanglement wedge of an evaporating black hole, J. High Energy Phys. 12 (2019) 063.

[14] G. Penington, Entanglement wedge reconstruction and the information paradox, J. High Energy Phys. 09 (2020) 002.

[15] A. Almheiri, R. Mahajan, J. Maldacena, and Y. Zhao, The page curve of Hawking radiation from semiclassical geometry, J. High Energy Phys. 03 (2020) 149.

[16] A. R. Brown, D. A. Roberts, L. Susskind, B. Swingle, and Y. Zhao, Holographic Complexity Equals Bulk Action?, Phys. Rev. Lett. 116, 191301 (2016).
[17] A. R. Brown, D. A. Roberts, L. Susskind, B. Swingle, and Y. Zhao, Complexity, action, and black holes, Phys. Rev. D 93, 086006 (2016).

[18] L. Lehner, R. C. Myers, E. Poisson, and R. D. Sorkin, Gravitational action with null boundaries, Phys. Rev. D 94, 084046 (2016).

[19] R. G. Cai, S. M. Ruan, S. J. Wang, R. Q. Yang, and R. H. Peng, Action growth for AdS black holes, J. High Energy Phys. 09 (2016) 161.

[20] S. Chapman, H. Marrochio, and R. C. Myers, Complexity of formation in holography, J. High Energy Phys. 01 (2017) 062.

[21] D. Carmi, S. Chapman, H. Marrochio, R. C. Myers, and S. Sugishita, On the time dependence of holographic complexity, J. High Energy Phys. 11 (2017) 188.

[22] P. Braccia, A. L. Cotrone, and E. Tonni, Complexity in the presence of a boundary, J. High Energy Phys. 02 (2020) 051.

[23] J. L. F. Barbon and E. Rabinovici, Holographic complexity and spacetime singularities, J. High Energy Phys. 01 (2016) 084.

[24] R. Auzzi, S. Baiguera, and G. Nardelli, Volume and complexity for warped AdS black holes, J. High Energy Phys. 06 (2018) 063.

[25] R. Auzzi, S. Baiguera, M. Grassi, G. Nardelli, and N. Zenoni, Complexity and action for warped AdS black holes, J. High Energy Phys. 09 (2018) 013.

[26] M. Alishahiha, Holographic complexity, Phys. Rev. D 92, 126009 (2015).

[27] D. Carmi, R. C. Myers, and P. Rath, Comments on holographic complexity, J. High Energy Phys. 03 (2017) 118.

[28] O. Ben-Ami and D. Carmi, On volumes of subregions in holography and complexity, J. High Energy Phys. 11 (2016) 129.

[29] R. Abt, J. Erdmenger, H. Hinrichsen, C. M. MelbyThompson, R. Meyer, C. Northe, and I. A. Reyes, Topological complexity in $\mathrm{AdS}_{3} / \mathrm{CFT}_{2}$, Fortschr. Phys. 66, 1800034 (2018).

[30] C. A. Agón, M. Headrick, and B. Swingle, Subsystem complexity and holography, J. High Energy Phys. 02 (2019) 145. 
[31] M. Alishahiha, K. Babaei Velni, and M. R. Mohammadi Mozaffar, Black hole subregion action and complexity, Phys. Rev. D 99, 126016 (2019).

[32] E. Caceres, J. Couch, S. Eccles, and W. Fischler, Holographic Purification Complexity, Phys. Rev. D 99, 086016 (2019).

[33] R. Auzzi, S. Baiguera, A. Mitra, G. Nardelli, and N. Zenoni, Subsystem complexity in warped AdS, J. High Energy Phys. 09 (2019) 114.

[34] B. Chen, W. M. Li, R. Q. Yang, C. Y. Zhang, and S. J. Zhang, Holographic subregion complexity under a thermal quench, J. High Energy Phys. 07 (2018) 034.

[35] R. Auzzi, G. Nardelli, F. I. Schaposnik Massolo, G. Tallarita, and N. Zenoni, On volume subregion complexity in Vaidya spacetime, J. High Energy Phys. 11 (2019) 098.

[36] R. Auzzi, S. Baiguera, A. Legramandi, G. Nardelli, P. Roy, and N. Zenoni, On subregion action complexity in $\mathrm{AdS}_{3}$ and in the BTZ black hole, J. High Energy Phys. 01 (2020) 066.

[37] E. Caceres, S. Chapman, J. D. Couch, J. P. Hernandez, R. C. Myers, and S. M. Ruan, Complexity of mixed states in QFT and holography, J. High Energy Phys. 03 (2020) 012.

[38] J. Hernandez, R. C. Myers, and S. M. Ruan, Quantum extremal islands made easy, Part III: Complexity on the brane, J. High Energy Phys. 02 (2021) 173.

[39] G. Di Giulio and E. Tonni, Complexity of mixed Gaussian states from Fisher information geometry, J. High Energy Phys. 12 (2020) 101.

[40] R. Jefferson and R. C. Myers, Circuit complexity in quantum field theory, J. High Energy Phys. 10 (2017) 107.

[41] S. Chapman, M. P. Heller, H. Marrochio, and F. Pastawski, Toward a Definition of Complexity for Quantum Field Theory States, Phys. Rev. Lett. 120, 121602 (2018).

[42] K. Hashimoto, N. Iizuka, and S. Sugishita, Time evolution of complexity in Abelian gauge theories, Phys. Rev. D 96, 126001 (2017).

[43] H. A. Camargo, P. Caputa, D. Das, M. P. Heller, and R. Jefferson, Complexity as a Novel Probe Of Quantum Quenches: Universal Scalings and Purifications, Phys. Rev. Lett. 122, 081601 (2019).

[44] J. M. Magán, Black holes, complexity and quantum chaos, J. High Energy Phys. 09 (2018) 043.

[45] P. Caputa and J. M. Magan, Quantum Computation as Gravity, Phys. Rev. Lett. 122, 231302 (2019).

[46] P. Bueno, J. M. Magan, and C. S. Shahbazi, Complexity measures in QFT and constrained geometric actions, arXiv:1908.03577.

[47] S. Chapman, J. Eisert, L. Hackl, M. P. Heller, R. Jefferson, H. Marrochio, and R. C. Myers, Complexity and entanglement for thermofield double states, SciPost Phys. 6, 034 (2019).

[48] R. Khan, C. Krishnan, and S. Sharma, Circuit complexity in fermionic field theory, Phys. Rev. D 98, 126001 (2018).

[49] L. Hackl and R. C. Myers, Circuit complexity for free fermions, J. High Energy Phys. 07 (2018) 139.
[50] M. Doroudiani, A. Naseh, and R. Pirmoradian, Complexity for charged thermofield double states, J. High Energy Phys. 01 (2020) 120.

[51] V. Balasubramanian, M. Decross, A. Kar, and O. Parrikar, Quantum complexity of time evolution with chaotic Hamiltonians, J. High Energy Phys. 01 (2020) 134.

[52] M. Flory and M. P. Heller, Geometry of complexity in conformal field theory, Phys. Rev. Research 2, 043438 (2020).

[53] M. Flory and M. P. Heller, Conformal field theory complexity from Euler-Arnold equations, J. High Energy Phys. 12 (2020) 091.

[54] A. R. Brown, L. Susskind, and Y. Zhao, Quantum complexity and negative curvature, Phys. Rev. D 95, 045010 (2017).

[55] A. R. Brown and L. Susskind, Second law of quantum complexity, Phys. Rev. D 97, 086015 (2018).

[56] A. Bernamonti, F. Galli, J. Hernandez, R. C. Myers, S. M. Ruan, and J. Simón, First Law of Holographic Complexity, Phys. Rev. Lett. 123, 081601 (2019).

[57] A. Bernamonti, F. Galli, J. Hernandez, R. C. Myers, S. M. Ruan, and J. Simón, Aspects of the first law of complexity, arXiv:2002.05779.

[58] D. V. Anosov, Geodesic flows on closed Riemannian manifolds with negative curvature, Proc. Steklov Inst. Mathematics 90, 1 (1967).

[59] L. Susskind and Y. Zhao, Switchbacks and the bridge to nowhere, arXiv:1408.2823.

[60] A. R. Brown and L. Susskind, Complexity geometry of a single qubit, Phys. Rev. D 100, 046020 (2019).

[61] B. O'Neill, The fundamental equations of a submersion, Michigan Math. J. 13, 459 (1966).

[62] B. O’Neill, Submersions and geodesics, Duke Math. J. 34, 363 (1967).

[63] J. Milnor, Curvatures of left invariant metrics on lie groups, Adv. Math. 21, 293 (1976).

[64] V. I. Arnold, Mathematical Methods of Classical Mechanics (Springer Verlag, Berlin, 1989).

[65] P. Petersen, Riemannian Geometry (Springer, New York, 2016).

[66] J. M. Lee, Riemannian Manifolds-An Introduction to Curvature (Springer, New York, 1998).

[67] K. Burns and M. Gerber, Real analytic Bernoulli geodesic flows on $S^{2}$, Ergodic Theory Dynam. Syst. 9, 27 (1989).

[68] R. J. Caginalp and S. Leutheusser, Complexity in One- and Two-Qubit Systems, arXiv:2010.15099.

[69] M. A. Nielsen and I. L. Chuang, Quantum Computation and Quantum Information (Cambridge University Press, Cambridge, England, 2010).

[70] L. Susskind, Black holes at exp-time, arXiv:2006.01280.

[71] A. L. Besse, Einstein Manifolds (Springer, New York, 1987).

[72] E. Poisson, A Relativist's Toolkit: The Mathematics of Black-Hole Mechanics, 10.1017/CBO9780511606601.

[73] S.M. Carroll, Spacetime and Geometry (Cambridge University Press, Cambridge, England, 2004). 